\title{
Functional antagonism between E2F family members
}

\author{
Maxim V. Frolov, ${ }^{1,4}$ David S. Huen, ${ }^{2,3,4}$ Olivier Stevaux, ${ }^{1}$ Dessislava Dimova, ${ }^{1}$ \\ Kristi Balczarek-Strang, ${ }^{1}$ Mark Elsdon, ${ }^{2}$ and Nicholas J. Dyson ${ }^{1,5}$ \\ ${ }^{1}$ Massachusetts General Hospital Cancer Center, Charlestown, Massachusetts 02129, USA; ${ }^{2}$ Cancer Research Campaign, \\ Department of Oncology, University of Cambridge CB2 2XY, UK ${ }^{3}$ Department of Genetics, University of Cambridge \\ CB2 3EH, UK
}

E2F is a heterogenous transcription factor and its role in cell cycle control results from the integrated activities of many different E2F family members. Unlike mammalian cells, that have a large number of E2F-related genes, the Drosophila genome encodes just two E2F genes, de2f1 and de2f2. Here we show that de2f1 and de2f2 provide different elements of E2F regulation and that they have opposing functions during Drosophila development. dE2F1 and dE2F2 both heterodimerize with dDP and bind to the promoters of E2F-regulated genes in vivo. dE2F1 is a potent activator of transcription, and the loss of $d e 2 f 1$ results in the reduced expression of E2F-regulated genes. In contrast, $\mathrm{dE} 2 \mathrm{~F} 2$ represses the transcription of E2F reporters and the loss of $d e 2 f 2$ function results in increased and expanded patterns of gene expression. The loss of de2f1 function has previously been reported to compromise cell proliferation. de $2 f 1$ mutant embryos have reduced expression of E2F-regulated genes, low levels of DNA synthesis, and hatch to give slow-growing larvae. We find that these defects are due in large part to the unchecked activity of $\mathrm{dE2F} 2$, since they can be suppressed by mutation of $d e 2 f 2$. Examination of eye discs from de2f1; de2f2 double-mutant animals reveals that relatively normal patterns of DNA synthesis can occur in the absence of both E2F proteins. This study shows how repressor and activator E2Fs are used to pattern transcription and how the net effect of E2F on cell proliferation results from the interplay between two types of E2F complexes that have antagonistic functions.

[Key Words: E2F; cell cycle; Drosophila]

Received April 13, 2001; revised version accepted June 18, 2001.

The E2F transcription factor plays an important role in the control of cell proliferation. E2F-dependent transcription is modulated during cell cycle progression from $\mathrm{G}_{1}$ to $\mathrm{S}$-phase and results obtained from a variety of different experimental systems show that the levels of E2F are rate-limiting for the $\mathrm{G}_{1} / \mathrm{S}$ transition. The overexpression of E2F genes is sufficient to drive quiescent cells into S-phase (Johnson et al. 1993; Asano et al. 1996; Du et al. 1996b; Lukas et al. 1996). In contrast, molecules that inhibit E2F-dependent transcription lead to the accumulation of cells in $\mathrm{G}_{1}$ (Dobrowolski et al. 1994; Sellers et al. 1995; Ishizaki et al. 1996; Wu et al. 1996). Consistent with these observations, E2F genes behave as oncogenes when overexpressed, and mutations that deregulate E2F activity are found in many tumor cells (for reviews, see Dyson 1998; Helin 1998; Nevins 1998).

E2F-binding sites allow gene transcription to be coupled with cell cycle position. E2F-regulated promoters are repressed in quiescent cells and induced as cells enter S-phase. E2F binding sites are thought to act as

\footnotetext{
${ }^{4}$ These authors contributed equally to this work.

${ }^{5}$ Corresponding author.

E-MAIL dyson@helix.mgh.harvard.edu; FAX (617) 726-7808.

Article and publication are at http://www.genesdev.org/cgi/doi/10.1101/ gad. 903901.
}

repressor elements in quiescent cells by recruiting $\mathrm{pRB}$ (or pRB-related proteins) and pRB-associated proteins (Dyson 1998; Harbour and Dean 2000). pRB has been proposed to block transcription in three general ways: by binding to the transcription activation domain of E2F and blocking its activity (Flemington et al. 1993; Helin et al. 1993), by inhibiting the activity of the basal transcription machinery (Ross et al. 1999), and by recruiting complexes that affect chromatin structure (Sellers et al. 1995; Brehm et al. 1998; Luo et al. 1998; Magnaghi-Jaulin et al. 1998; Meloni et al. 1999; Ross et al. 2001). As cells enter S-phase, these interactions are disrupted and transcriptional repression is relieved. Some studies have suggested that expression of E2F target genes results primarily from de-repression of the promoter; others have suggested that E2F-binding sites also mediate transcriptional activation in S- or $\mathrm{G}_{2}$-phase (Means et al. 1992; Wade et al. 1992; Lam and Watson 1993; Hsiao et al. 1994; Johnson et al. 1994; Neuman et al. 1994; Lam et al. 1995; Fry et al. 1997; Yamaguchi et al. 1997). The precise number of E2F targets is not known and recent studies suggest that E2F may regulate the expression of many genes that are involved in diverse aspects of cellular function (Muller et al. 2001). The best-known targets of E2F encode proteins that are critical for cell cycle pro- 
gression and DNA synthesis. These include cell-cycle regulators, such as cyclin $\mathrm{E}(\mathrm{CycE})$, cyclin $\mathrm{A}(\mathrm{CycA})$, cdc2, cdc25, Myc, B-myb, and products that are required for DNA replication, such as large subunit of DNA polymerase $\alpha$, ribonucleotide reductase, proliferating cellular nuclear antigen (PCNA), dehydrofolate reductase (DHFR), ORC proteins, and minichromosome maintenance proteins (MCMs) (Helin 1998).

Studies of E2F in mammalian cells are hampered by the complexity of E2F. The term E2F refers to the collective activity of many different proteins. The basic unit of E2F is a heterodimer, composed of an E2F and a DP subunit. Six E2F genes and two DP genes have been studied, and recent surveys of the human genome raise the possibility that several additional family members may also exist (Aravind et al. 2001). Precisely how the functions of all of these proteins are integrated together is unclear. Biochemical experiments show that different types of E2F complexes have similar DNA-binding properties and, even in populations of synchronized tissue culture cells, many different E2F proteins can be found at a given promoter (Takahashi et al. 2000; Wells et al. 2000). E2F-1, E2F-2, E2F-3, E2F-4, and E2F-5 are each capable of activating transcription and can be repressed by pRB-family proteins. Nevertheless, there are several indications that individual E2Fs are tailored for distinct functions. E2F-1, E2F-2, and E2F-3, for example, are more potent transcriptional activators than E2F-4 and E2F-5 when these proteins are overexpressed. The expression of E2F-1, E2F-2, and E2F-3a is cell cycle regulated and the appearance of these proteins at E2F regulated promoters generally correlates with gene expression. Conversely, E2F-4 appears to be the predominant E2F at times when E2F-regulated promoters are repressed (Sardet et al. 1995; Lukas et al. 1996; Moberg et al. 1996; DeGregori et al. 1997; Muller et al. 1997; Verona et al. 1997; Adams et al. 2000; Leone et al. 2000).

The phenotypes resulting from the targeted inactivation of E2F genes in mice confirm that individual E2F genes do have some unique functions. $E 2 f-1^{-/-}$mice have defects in thymocyte development and are tumor-prone. The loss of E2f-5 does not cause any defects in cell proliferation (Lindeman et al. 1998). Instead, mice die due to hydrocephalus caused by excessive secretion of cerebral spinal fluid, an alteration caused by abnormalities in cell differentiation. Mice deficient for E2f-4 display multiple developmental defects and die due to increased susceptibility to opportunistic infections (Humbert et al. 2000a; Rempel et al. 2000). Although E2F-4 accounts for the majority of the endogenous E2F DNA-binding activity in many cell types, E2F-4 is fully dispensable for the cell cycle arrest or proliferation and mutation of E2F-4 gave no discernable effect on transcription of known E2F target genes (Humbert et al. 2000a; Rempel et al. 2000). Unlike the mutant phenotypes described above, E2f-3-1cell lines show reduced rate of proliferation and deregulation in the expression of E2F responsive genes (Humbert et al. 2000b). Interpretation of all of these phenotypes is complicated by the presence of multiple family members with related or overlapping functions, which might mask or modify the effects of removing an individual form of E2F. For example, studies of E2f-4; E2f-5 double-mutant MEFs show that these forms of E2F have a redundant function that is required for p16-induced cell cycle arrest (Gaubatz et al. 2000). Although E2F is considered to be a critical cell cycle regulator, none of the E2F mouse knockouts that have been reported to date to have resulted in a generalized block to cell proliferation in vivo. This discrepancy is thought to be due to functional overlap or compensation between E2F proteins, such that most cell types contain several different E2F complexes that can provide the E2F functions essential for cell proliferation.

Here we describe experiments using Drosophila as a model system to study E2F function. The Drosophila genome contains two E2F genes and one DP gene (Dynlacht et al. 1994; Ohtani and Nevins 1994; Hao et al. 1995; Sawado et al. 1998b) and hence the issues of functional redundancy and overlap are likely to be less severe. Previous studies have shown that dE2F1 and dDP share many biochemical and functional similarities with their mammalian counterparts. These proteins heterodimerize, bind to consensus E2F sites, and cooperate to activate transcription when overexpressed (Dynlacht et al. 1994). High levels of dE2F1/dDP drive quiescent cells into S-phase and stimulate apoptosis (Asano et al. 1996; Du et al. 1996b). dE2F1 and dDP associate with an RB-related protein (RBF) that represses dE2F1-dependent transcription in tissue culture cells and in vivo and blocks dE2F1-induced proliferation (Du et al. 1996a).

de $2 f 1$ provides an essential function in vivo. de2f1 mutants are defective during embryogenesis (Duronio et al. 1995), show a significant delay in larval growth, and fail to complete larval development (Royzman et al. 1997; Du 2000). de2f1 mutant embryos lack a $G_{1} / S$ transcriptional program that normally accompanies S-phase entry and loss of de2f1 leads to an almost complete cessation of DNA synthesis by stage 13 of embryogenesis (Duronio et al. 1995; Royzman et al. 1997). Analysis of de2f1 mutant clones in imaginal discs confirmed that dE2F1 is required for normal cell proliferation (Brook et al. 1996; Neufeld et al. 1998) and suggested that E2F also acts in postmitotic cells (Brook et al. 1996). Studies of partial loss-of-function alleles in the ovary have implicated E2F in the shut off of DNA synthesis in follicle cells and have shown that de2f1 is required in this cell type for amplification of chorion gene clusters (Royzman et al. 1997).

$d D P$ mutant embryos resemble de2f1 mutants in lacking a $G_{1} / S$ transcriptional program, but the effects of $d D P$ mutation on the expression of genes that are normally expressed at $\mathrm{G}_{1} / \mathrm{S}$ varies and depends on the target gene examined (Royzman et al. 1997; Duronio et al. 1998). Examination of $d D P$ mutant clones and specific alleles of $d D P$ shows that $\mathrm{dDP}$ is required during oogenesis (Myster et al. 2000) and that it is required for the shut off of DNA synthesis in follicle cells (Royzman et al. 1999). However, the patterns of DNA synthesis and cell proliferation are not severely affected in $d D P$ mutant embryos or $d D P$ mutant larvae, indicating that the functions of $\mathrm{dE} 2 \mathrm{~F} 1$ and $\mathrm{dDP}$ are not equivalent. 
The differences between the de2f1 and $d D P$ mutant phenotypes have led to speculation that dE2F1 might have functions that are independent of dDP, or alternatively that dDP might have functions that are independent of dE2F1 (Royzman et al. 1997; Duronio et al. 1998). One gene that is likely to have an impact on these phenotypes is de2f2, a de2f1-related gene that was uncovered by direct sequencing of transcription units within the 39B-D cytological region (D. Huen et al., unpubl.), in a two-hybrid screen using RBF1 as bait (W. Du and N. Dyson, unpubl.), and in the Drosophila genome project (Sawado et al. 1998b; Rubin et al. 2000). Biochemical experiments have shown that dE2F2 can cooperate with dDP to generate specific DNA-binding activity on a consensus E2F-binding site and that a dE2F2 expression plasmid weakly repressed the transcription of an E2F-reporter construct when transfected into the Drosophila Kc cell line (Sawado et al. 1998b). Nothing is known about the normal function of dE2F2. Here we show that $\mathrm{dE} 2 \mathrm{~F} 2$ is a physiological partner for $\mathrm{dDP}$ and RBF and that dE2F2 acts antagonistically to dE2F1 during Drosophila development. We find that mutations in de2f2 relieve the block to DNA synthesis and larval development that is caused by mutation of de2f1. These results show that E2F-control of cell proliferation results from the interplay between two types of E2F complexes that have opposing activities and antagonistic functions.

\section{Results}

The endogenous dE2F2 protein associates with $d D P$ and $R B F$

As an initial step in the characterization of dE2F2 we generated dE2F2-specific antibodies and determined whether dE2F2 associates with dDP and RBF in vivo. SL2 cell extracts were immunoprecipitated in parallel using anti-dDP and anti-dE2F2 mouse monoclonal antibodies coupled to beads. After washing, the immune complexes were separated by SDS-PAGE and Western blots were probed with anti-RBF, anti-dE2F2, and anti-dDP antibodies (Fig. 1). Both RBF and dDP were detected in the antidE2F2 immunoprecipitates. No signal was observed using a negative control antibody or without cell extracts. In the same way, dE2F2 was readily detected in anti-dDP immune complexes. Immunoprecipitation experiments were repeated using embryo extracts and gave similar results (Fig. 1). We conclude that dE2F2 associates with $\mathrm{dDP}$ and RBF under normal physiological conditions.

\section{dE2F2 represses transcription in Drosophila cell culture}

To compare the transcriptional properties of $\mathrm{dE} 2 \mathrm{~F} 2$ and $\mathrm{dE} 2 \mathrm{~F} 1$, both cDNAs were cloned into an expression vector and tagged with a myc epitope on the $\mathrm{N}$ terminus. The use of a common epitope enabled us to examine both the activities and the levels of these proteins in transient transfection experiments. Expression plasmids were cotransfected into Drosophila SL2 cells together
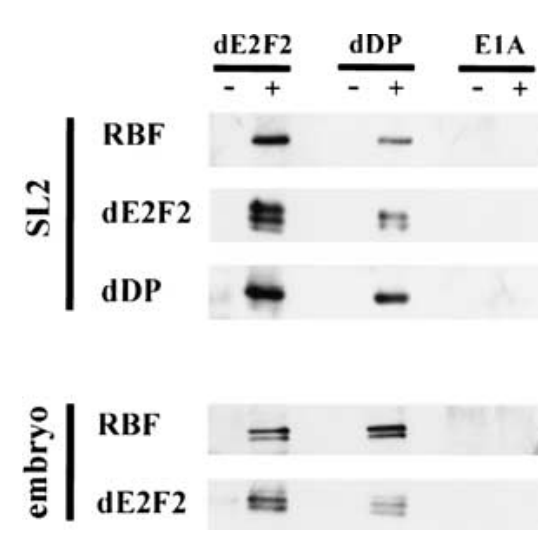

Figure 1. $\mathrm{dE} 2 \mathrm{~F} 2$ associates with $\mathrm{dDP}$ and $\mathrm{RBF}$ in vivo. Beadcoupled anti-dE2F2, anti-dDP, or anti-E1A antibodies were used to immunoprecipitate complexes from SL2 cell or embryonic extracts $(+)$. Associated proteins were detected by Western blot analysis with antibodies specific for RBF, dE2F2, or dDP. Because dE2F2 and dDP migrate in the vicinity of IgG heavy chain, additional controls were included in which the coupled beads were incubated in lysis buffer alone (minus sign). RBF migrates as a doublet in embryonic extracts.

with a reporter plasmid in which the luciferase gene is fused to the promoter region from -168 to +23 of PCNA gene containing two canonical E2F sites (Yamaguchi et al. 1995). The contribution of endogenous E2F activity on the luciferase reporter was estimated when the cells were transfected with an empty vector.

Equal amounts of dE2F1 and dE2F2 plasmids were separately transfected into Drosophila SL2 cells and the luciferase levels produced from the reporter plasmid were measured in triplicate samples (Fig. 2A). Luciferase activity was stimulated upon transfection of dE2F1, in a manner that was dependent on the E2F-binding sites in the promoter, as described previously (Dynlacht et al. 1994). In contrast, transfection of the dE2F2 plasmid repressed the reporter construct by up to 10-fold (Fig. 2A). The repressive activity of dE2F2 is much greater than the twofold effect reported previously (Sawado et al. 1998b). The greater level of dE2F2-mediated repression described here may be due to the use of different cell lines or to higher levels of expression provided by the ie 4 baculovirus promoter. Because dE2F1 and dE2F2 had opposite effects on transcription we performed competition experiments in which a constant amount of dE2F1 was challenged with increasing amounts of dE2F2 and a constant amount of dE2F2 was challenged with increasing amounts of dE2F1. As might be expected, the degree of activation or repression depended on the ratio of the expression constructs. Interestingly however, Western blot analysis of the transfected cells showed that a barely detectable quantity of dE2F1 protein was sufficient to overcome the repression by dE2F2; whereas even a 20 fold excess of dE2F2 could only partly block transcriptional activation by dE2F1 (Fig. 2B,C).

Although these results suggest that dE2F1 and dE2F2 have opposing effects on the PCNA promoter, an alternative, albeit trivial, explanation for these results might 

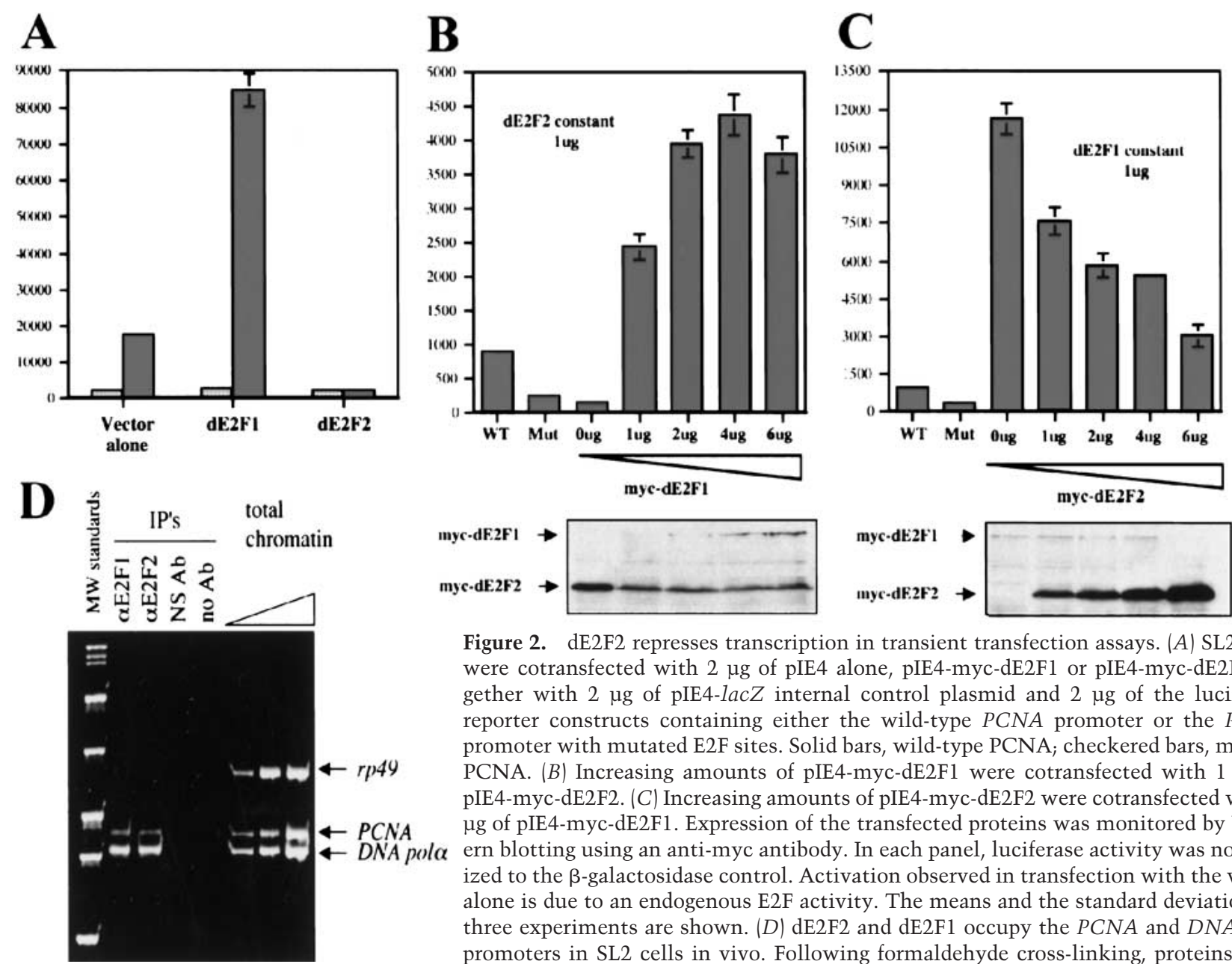

Figure 2. dE2F2 represses transcription in transient transfection assays. (A) SL2 cells were cotransfected with $2 \mu$ g of pIE4 alone, pIE4-myc-dE2F1 or pIE4-myc-dE2F2 together with $2 \mu \mathrm{g}$ of pIE4-lacZ internal control plasmid and $2 \mu \mathrm{g}$ of the luciferase reporter constructs containing either the wild-type PCNA promoter or the PCNA promoter with mutated E2F sites. Solid bars, wild-type PCNA; checkered bars, mutant PCNA. (B) Increasing amounts of pIE4-myc-dE2F1 were cotransfected with $1 \mu \mathrm{g}$ of pIE4-myc-dE2F2. (C) Increasing amounts of pIE4-myc-dE2F2 were cotransfected with 1 $\mu \mathrm{g}$ of pIE4-myc-dE2F1. Expression of the transfected proteins was monitored by Western blotting using an anti-myc antibody. In each panel, luciferase activity was normalized to the $\beta$-galactosidase control. Activation observed in transfection with the vector alone is due to an endogenous E2F activity. The means and the standard deviations of three experiments are shown. $(D) \mathrm{dE} 2 \mathrm{~F} 2$ and dE2F1 occupy the PCNA and DNA pol $\alpha$ promoters in SL2 cells in vivo. Following formaldehyde cross-linking, proteins were immunoprecipitated with anti-dE2F2 ( $\alpha$-E2F2 IP), with anti-dE2F1 ( $\alpha$-E2F1 IP), or nonspecific (NS IP) antibodies, and the associated chromatin fragments were used as a template for PCR amplification with primers specific for DNA pol $\alpha$, PCNA, and rp49 promoter sequences. Three dilutions of total chromatin (triangle) were amplified with the same set of primers.

arise if $P C N A$ were an exclusive target for $\mathrm{dE} 2 \mathrm{~F} 1$. If this were the case, the failure of dE2F2 to activate the reporter might be misleading and a consequence using a promoter that is not normally regulated by dE2F2. To test this possibility, we used a chromatin immunoprecipitation assay to enrich for DNA fragments occupied by dE2F1 or dE2F2 in vivo. Proteins bound to DNA in SL2 cells were cross-linked using formaldehyde, chromatin was isolated, and E2F proteins was immunoprecipitated using antibodies specific for either dE2F1 or dE2F2 antibodies. The presence of E2F-regulated promoters was detected by PCR using gene-specific primers. As shown in Figure 2D, PCNA promoter sequences were selectively enriched in the anti-dE2F1 and anti-dE2F2 immunoprecipitates relative to control fragments. No enrichment was observed using unrelated antibodies to control for nonspecific DNA-binding activity, and a similar enrichment was seen using primers to a second $\mathrm{dE} 2 \mathrm{~F} 1$-in-

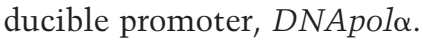

We conclude that $\mathrm{dE} 2 \mathrm{~F} 2$ is normally present at promoters that have previously been shown to be induced by $\mathrm{dE} 2 \mathrm{~F} 1$. Whereas $\mathrm{dE} 2 \mathrm{~F} 1$ expression activates transcrip- tion from these promoters, $\mathrm{dE} 2 \mathrm{~F} 2$ expression represses transcription. However, low levels of $\mathrm{dE} 2 \mathrm{~F} 1$ readily overcome the repressor activity of $\mathrm{dE} 2 \mathrm{~F} 2$.

\section{Interactions between de $2 \mathrm{f} 1$ and de $2 \mathrm{f} 2$ in transgenic animals}

To test the physiological relevance of these observations we asked whether similar interactions between dE2F1 and $\mathrm{dE} 2 \mathrm{~F} 2$ could be detected in vivo. Genetic interactions between $\mathrm{dE} 2 \mathrm{~F} 1, \mathrm{dDP}$, and RBF have been examined previously using pGMR transgenes that allow ectopic expression to be targeted specifically to developing eye. Unfortunately, GMR-de2f2 transgenes gave no discernable eye phenotype. Immunostaining and Western blot analysis of eye discs showed that, unlike the previously described transgenes, expressing $\mathrm{dE} 2 \mathrm{~F} 1, \mathrm{dDP}$, and RBF the GMR-de2f2 transgenes were insufficient to increase dE2F2 levels above the endogenous protein. This suggests that endogenous $\mathrm{dE} 2 \mathrm{~F} 2$ is expressed at high level in the eye disc. An affinity-purified rabbit polyclonal antibody gave a strong ubiquitous staining throughout the 
disc (Fig. 3A). The levels of dE2F2 do not appear to vary greatly within the disc, in contrast to dE2F1 that has a dynamic pattern of expression (see Asano et al. 1996; Brook et al. 1996).

The use of UAS-de2f2 and GMR-GAL4 transgenes provided a higher level of dE2F2-expression. Immunostaining of eye discs from UAS-de2f2; GMR-GAL4 larvae confirmed that the ectopic expression of dE2F2 begins as cells are entering the morphogenetic furrow and is maintained posterior to the furrow in differentiating cells (Fig. 3B). Western blot analysis showed that dE2F2 is produced at 5- to 10 -fold higher levels than the endogenous protein (Fig. 3B). Under scanning electron microscopy (SEM) the eyes of flies carrying one copy of UASde2f2 and GMR-GAL4 show severely abnormal eyes compared to the GMR-GAL4 control (Fig. 3C,D). These eyes were rough in appearance and ommatidia lacked their regular pattern. This phenotype was generated with three independent UAS-de2f2 transgenes, suggesting that the effects are not due to the particular site of the transgene insertion. The phenotype was enhanced when two copies of the UAS-de2f2 transgene were present indicating that the effects of dE2F2 were not saturated (Fig. $3 \mathrm{E})$. When the levels of $\mathrm{dE} 2 \mathrm{~F} 1$ were manipulated, we observed that eye phenotype induced by the de2f2 transgene was almost completely suppressed by elevated levels of dE2F1 (Fig. 3F). Conversely, the roughness of the eye was slightly enhanced in flies heterozygous for $d e 2 f 1$ and carrying either the de2f1 $1^{r m 729}$ or $d e 2 f 1^{7172}$ mutant alleles (Fig. 3G).

These eye interactions are consistent with results of the transfection assays; both types of experiments raise the possibility that $\mathrm{dE} 2 \mathrm{~F} 1$ and $\mathrm{dE} 2 \mathrm{~F} 2$ might have opposing activities. However, both sets of results are drawn from settings in which dE2F1 and/or dE2F2 are expressed at abnormal levels and it is uncertain that these effects accurately reflect the activities of the endogenous proteins. To test this we generated mutant alleles of de2f2 and compared the phenotypes of de2f2 mutant and de2f1; de2f2 double-mutant animals.

\section{Generation of de2f2 mutant allele}

The de2f2 gene is located at 39B2-3 on the left arm of the chromosome 2 and, to date, no mutations in de2f2 have been reported. An orphan P-element insertion line, 1(2)k16403, was found in which the $P$ element is inserted into the adjacent gene, MPP6, $667 \mathrm{bp}$ upstream of the $5^{\prime}$ end of de2f2. To generate a deletion of de2f2, which does not disrupt $M P P 6$, a two-step male recombination approach was utilized (Preston et al. 1996; for details and a map, see Materials and Methods). Briefly, in the first round, two deletions, de $2 f 2^{G 5.1}$ and $d e 2 f 2^{Q 3.1}$, were generated, which extend distally from the $P$-element insertion site. The de2f2 $2^{\text {3.1 }}$ chromosome, carrying the smaller of the two deletions, was used in a second round
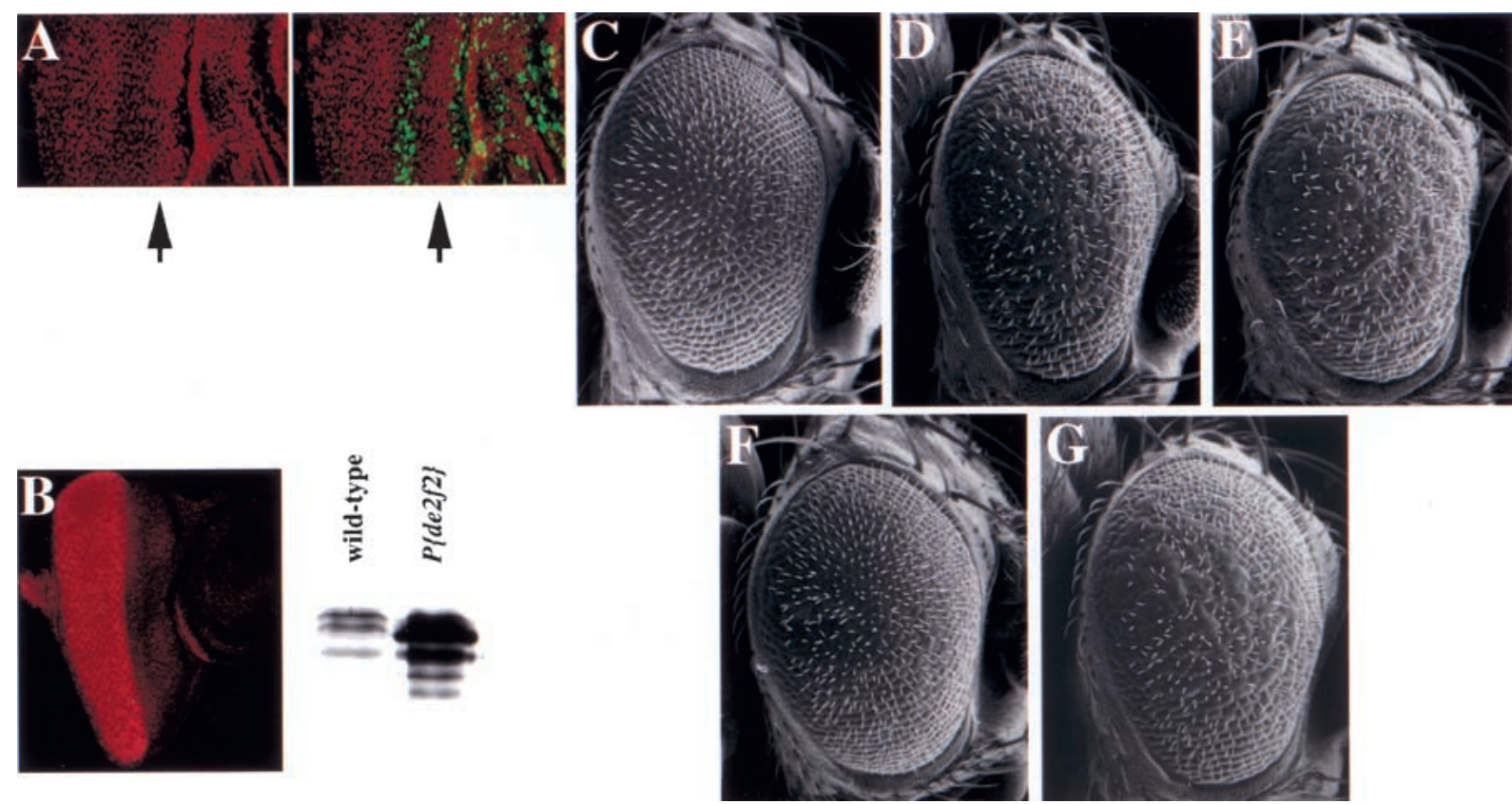

Figure 3. Ectopic expression of dE2F2 can induce a rough-eye phenotype that is suppressed by overexpression of $\mathrm{dE} 2 \mathrm{~F} 1$. $(A) \mathrm{dE} 2 \mathrm{~F} 2$ is ubiquitously expressed in wild-type imaginal disc, with no overt change in $\mathrm{G}_{1}$-phase cells of the morphogenetic furrow (arrow) or in S-phase cells. Eye discs were stained with anti-dE2F2 (red) and anti-BrdU (green) to identify the first and second mitotic waves. (B) dE2F2 expression is induced posterior to the morphogenetic furrow in GMR-GAL4/+; UAS-de2f2/+ animals. $(C-G)$ Scanning electron microscopy micrographs of Drosophila eyes. The ommatidial pattern of control flies, GMR-GAL4/+ $(C)$, is disrupted followed overexpression of dE2F2 in GMR-GAL4/+; UAS-de2f2/+ (D), and this effect is enhanced in GMR-GAL4/+; UAS-de2f2/UAS-de2f2 (E). This phenotype is strongly suppressed by overexpression of dE2F1 in GMR-GAL4/GMR-de2f1; UAS-de2f2/+ $(F)$ and slightly enhanced in flies heterozygous for de2f1 $1^{\text {rm729 }}$ allele, GMR-GAL4/+; UAS-de2f2/de2f $1^{r m 729}(G)$. 
of male recombination to generate a duplication event that restored the sequence of MPP6 but not de2f2 $\left(\right.$ de $\left.2 f 2^{76 Q 1}\right)$.

The following data strongly argue that $d e 2 f 2^{76 Q 1}$ represents a null allele for de2f2 and that the neighboring MPP6 gene is functional. Sequencing the de2f2 genomic region from the $d e 2 f 2^{76 Q 1}$ chromosome revealed that the de $2 f 2^{76 Q 1}$ deletion extends from a position 378 bp upstream of the de2f2 transcription start site to amino acid 5 of the coding sequence (for more details, see Materials and Methods). Hence this deletion removes de2f2 promoter sequences, the $5^{\prime}$ untranslated region, and the $5^{\prime}$ end of the dE2F2 ORF, including the presumptive start codon. The de2f2 ${ }^{76 Q 1}$ chromosome contains 273 bp upstream of the $M M P 6$ transcription start site. This is sufficient for MPP6 function since de2 $f 2^{76 Q 1}$ fully complements a lethal point mutation in MPP6, MPP6 ${ }^{U 39}$, in which the sixth amino acid is converted into a stop codon and, presumably, represents a null allele (D.S. Huen, unpubl.). In contrast, neither de2f2 $2^{\mathrm{3} .1}$ nor de2f2 ${ }^{G 5.1}$ can complement MPP6 ${ }^{U 39}$. Taken together, these data show that MPP6 is functional in the $d e 2 f 2^{76 Q 1}$ allele.

To confirm that the $d e 2 f 2^{76 Q 1}$ allele represents a null allele for de2f2, total RNA or protein extracts were prepared from second instar larvae trans-heterozygous for de2f2 $2^{761}$ and $d e 2 f 2^{G 5.1}$ alleles and compared to wildtype controls (for details of crosses, see Materials and Methods). Northern blot analysis using the full length de2f2 cDNA showed that de2f2 ${ }^{76 Q 1} / d e 2 f 2^{G 5.1}$ trans-heterozygous mutant larvae contained no detectable de2f2 transcripts (data not shown), and Western blot analysis with anti-dE2F2 antibodies showed that these animals contained no detectable dE2F2 protein (Fig. 4A).

Flies carrying the de2 $f 2^{76 Q 1}$ allele were crossed to various deletions removing de2f2. The de2f2 ${ }^{76 Q 1}$ allele was found to be semiviable over both deletions, de2f2 ${ }^{G 5.1}$ and de2f2 ${ }^{\text {3.1 }}$, as well as over the larger deficiencies $D f(2 L) T W 1$ and $D f(2 L)$ prA-14, that also remove de2f2. The number of trans-heterozygous escapers was variable for each combination of alleles and, moreover, was strongly affected by the genetic background. We observed trans-heterozygotes at frequencies that varied between $2 \%$ and $50 \%$ of the expected number. This variation suggests that animals deficient for dE2F2 activity are highly sensitive to heterozygosity at other loci in the genome.

de2 $f 2^{76 Q 1}$ homozygotes survived until adult stages at frequencies between $20 \%$ and $35 \%$ of the expected numbers. In addition to reduced viability, de2f2 null flies exhibit reduced fertility. Only $50 \%$ of eggs laid by de2f2 null females hatched compared to $95 \%$ for wild-type females. About $5 \%$ of the eggs deposited by de2f2 null females have fragile, thin chorions (data not shown; P. Cayirlioglu and B. Duronio, pers. comm.). de2f2 null males have significantly reduced fertility but did give progeny at low frequency when crossed to wild-type females. Curiously, normal progeny were recovered from crosses of de2f2 null males and females, indicating that animals can survive without any paternal, maternal, or

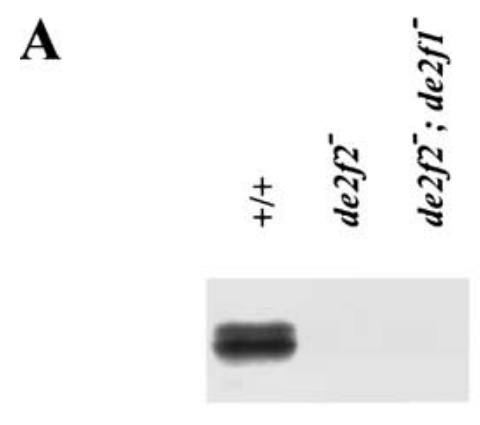

\section{B}

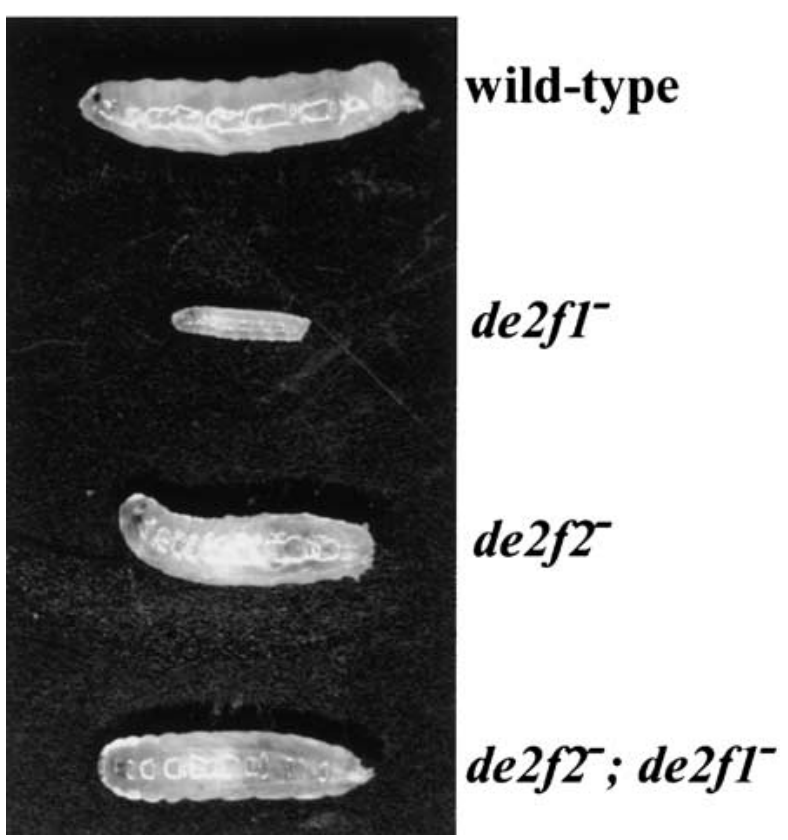

Figure 4. The loss of de2f2 suppresses larval growth defects caused by loss of de2f1. (A) Western blot analysis using polyclonal anti-dE2F2 antisera on extracts of de2f2 ${ }^{G 5.1} /$ de $f 2^{76 \mathrm{Q} 1}$ and de2f2 $2^{G 51} /$ de $2 f 2^{76 Q 1}$; de2f1 ${ }^{91} /$ de $f 1^{\text {rm729 }}$ larvae illustrates that no detectable amount of dE2F2 protein is synthesized from the de2f2 ${ }^{76 Q 1}$ allele. $(B)$ Larvae on the fifth day after egg laying of the following genotype Canton S (wild-type), de2f191/

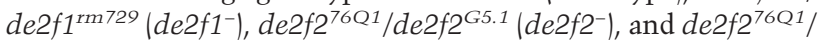

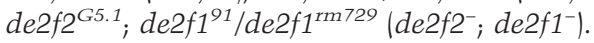

zygotic source of dE2F2. Thus, the loss of de2f2 results in reduced fertility and viability, but $\mathrm{dE} 2 \mathrm{~F} 2$ is not essential for animal development.

Loss of de2f2 suppresses phenotypes caused by loss of de $2 \mathrm{f} 1$

Studies in mammalian cells strongly indicate that several E2F family members share overlapping functions. If the Drosophila E2F proteins perform functions in vivo that are similar and overlapping, then de2f1; de2f2 double mutants would be predicted to be more severely affected than either the de2f1 or de2f2 single mutants. However, if $\mathrm{dE} 2 \mathrm{~F} 1$ and $\mathrm{dE} 2 \mathrm{~F} 2$ act antagonistically, as 
suggested above, then this result is unlikely. Although several outcomes are possible, the simplest possibility to consider is that the double mutant might be less severely affected than the single de2f1 or de2f2 mutants.

To answer this question, we generated animals transheterozygous for null alleles of dE2F2 (de2f2 ${ }^{76 Q 1}$ and de2f $\left.2^{G 5.1}\right)$ and dE2F1 (de2f1 ${ }^{91}$ and de2f1 $\left.{ }^{\text {rm729 }}\right)$. Both de2f1 alleles have been characterized previously and are considered to act as null alleles (Duronio et al. 1995; Royzman et al. 1997). Trans-heterozygous combinations of alleles were used to minimize the possible effects of any additional mutations that might be linked in cis. Embryos trans-heterozygous for de2f1 mutations lack the $G_{1} / S$ transcriptional program, show a dramatic reduction in DNA synthesis after stage 13, and hatch to give severely abnormal larvae (Duronio et al. 1995; Royzman et al. 1997; Du 2000). Five days after egg laying (AEL) de2f1 mutant larvae were much smaller than wild type (Fig. 4B). These animals failed to develop to the third larval instar and, as previously reported, contained severely underdeveloped imaginal discs and CNS.

Strikingly, the slow growth and abnormal larval development of the de2f1 mutant are almost completely suppressed by removing the dE2F2 activity. Trans-heterozygous double mutants, de2f276Q1/de2f2 ${ }^{G 5.1}$; de2f $f 1^{91}$ / de2 $f 1^{\text {rm729, }}$, developed normally without any significant delay in larval growth, reached pupal stage, and finally died as mid- or late pupae. The imaginal discs and CNS of de2f1; de2f2 double-mutant larvae were similar in development to wild-type primodia. The suppression of the de2f1 mutant phenotype is not restricted to a particular de2f1 allelic combination. Similar results were obtained when de $2 f 1^{r m 729}$ or de2f $f 1^{91}$ were placed over deficiency $D f(3 R) e-B S 2$ that uncovers de2f1.

de2f1 has previously been shown to be required for normal cell proliferation and DNA replication at several stages of Drosophila development. de2f1 mutant clones fail to proliferate in eye and wing imaginal discs (Brook et al. 1996; Neufeld et al. 1998). Surprisingly, in eye discs that lack both de2f1 and de2f2 the pattern of DNA synthesis is largely normal (Fig. 5). In wild-type or in mutant discs BrdU staining is localized primarily to the large area of asynchronously dividing cells in the first mitotic wave and in the synchronous second mitotic wave (Fig. $5 \mathrm{~A}, \mathrm{~B})$. Similar patterns were observed in de2f2 mutant discs (Fig. 5C). No BrdU incorporation was detected in the morphogenetic furrow, and even relatively long pulses of BrdU incorporation ( $2 \mathrm{~h}$ ) failed to reveal a significant number of ectopic S-phases posterior to the second mitotic wave in the double-mutant discs (Fig. 5D,E). Staining for ELAV, a marker of committed neuronal precursors, showed that the onset of neuronal differentiation was not severely perturbed by the absence of E2F proteins (Fig. 5F-H).

In examining large numbers of eye discs for these genotypes we observed that the intensity of label incorporated into the most intensely labeled nuclei during long-term BrdU pulses tended to be slightly reduced in de2f1; de2f2 double mutant relative to wild-type discs (Fig. 5D,E). However this reduction is considerably less
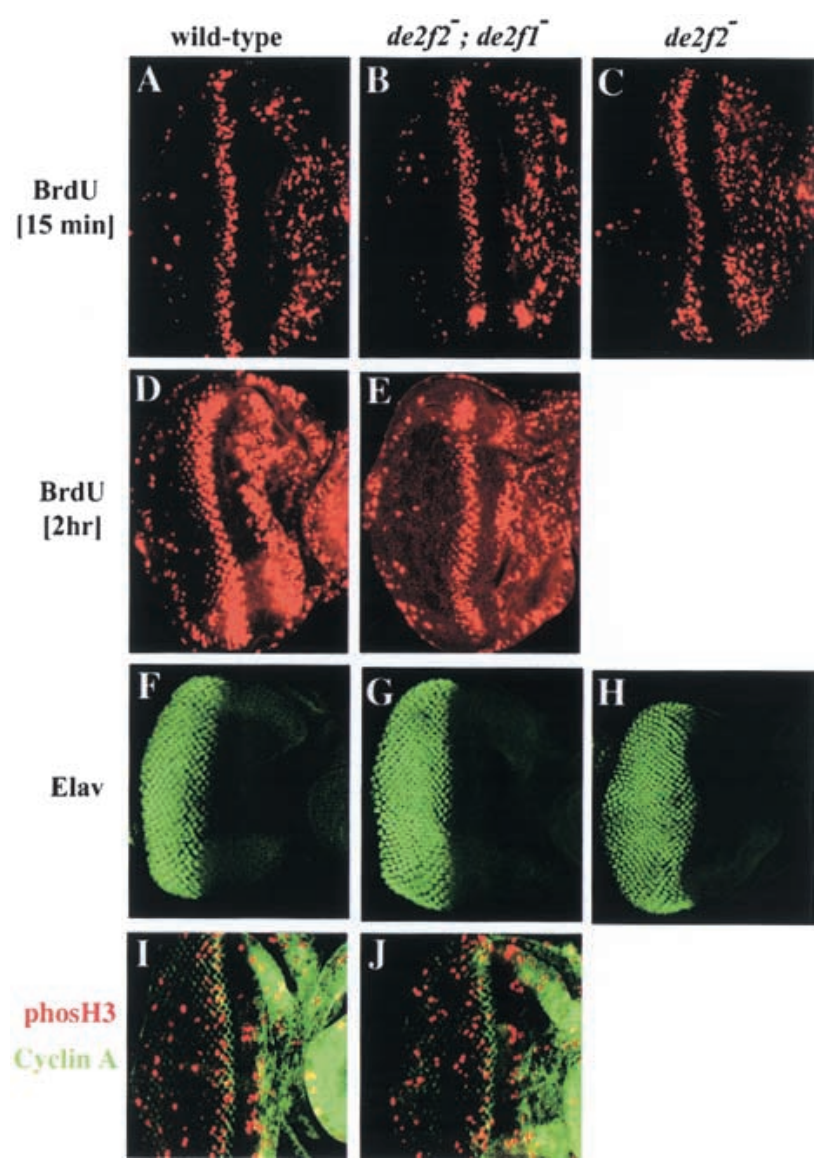

Figure 5. The patterns of DNA synthesis in eye discs lacking either dE2F2 or both dE2F1 and dE2F2 are similar to wild-type discs. Eye discs from third instar larvae are shown from wild-

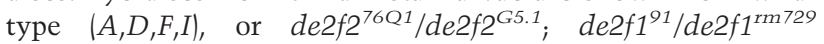
$(B, E, G, J)$ or de2f2 ${ }^{76 Q 1} / d e 2 f 2^{G 5.1}(C, H)$ animals. $(A-E)$ shows eye discs labeled with BrdU for $20 \mathrm{~min}(A-C)$ or $2 \mathrm{~h}(D-E)$. $(F-H)$ The onset of neuronal differentiation as revealed by staining for ELAV. $(I, J)$ The staining patterns for cyclin A (green), a marker of $\mathrm{G}_{2}$ cells, and phosH3 (red), a marker of mitotic cells. Note that the patterns of each of these markers are similar in discs of wild-type and double-mutant animals.

than the normal variation that occurs between discs of a single genotype. To further examine cell cycle progression in the absence of E2F regulation, de2f2; de2f1 double-mutant discs were double stained with anti-cyclin A and anti-phosphorylated histone $\mathrm{H} 3$ (phosH3) antibodies. Cyclin $A$ is commonly used as a $G_{2}$ marker because it is expressed in cells that have passed the $G_{1} / S$ transition and is degraded when cells enter mitosis (Knoblich and Lehner 1993). The phos H3 antibody preferentially stains M-phase cells (de Nooij et al. 1996). The patterns of Cyclin A and phosH3 staining in de2f2; de2f1 double-mutant eye discs (Fig. 5J) were indistinguishable from wild type (Fig. 5I). Thus, cells of the eye imaginal disc can proliferate asynchronously in the first mitotic wave, arrest synchronously in the morphogenetic furrow, re-enter the cell cycle synchronously in the second mitotic wave, permanently exit the cell cycle, and differentiate all in the absence of both dE2F1 and dE2F2. 
We conclude that the loss of $d e 2 f 2$ suppresses the larval growth, cell proliferation, and DNA-replication defects that are caused by the mutation of $d e 2 f 1$. This suppression confirms that $\mathrm{dE} 2 \mathrm{~F} 1$ and $\mathrm{dE} 2 \mathrm{~F} 2$ have antagonistic functions, at least during larval development. In theory, the effects of dE2F2 that are evident in the de2f1 mutant could be caused by an unusual dE2F2 activity that is independent of dDP. However, the loss of $d D P$ function behaves in a similar way to loss of de2f2 and suppresses the developmental defects of de2f1 mutant larvae (Fig. 6). Thus it is most likely that the severe defects seen in de2f1 mutant larvae require the action of $\mathrm{dE} 2 \mathrm{~F} 2 / \mathrm{dDP}$ heterodimers.

Deregulation of the expression of E2F target genes in the de2f2 single-mutant and $\operatorname{de} 2 \mathrm{f} 2 ; \operatorname{de} 2 \mathrm{f} 1$ double-mutant eye discs

The relatively normal patterns of cell proliferation in de2f1; de2f2 mutants are, at first glance, difficult to reconcile with the idea that E2F is a critical regulator of gene expression and cell proliferation. We therefore examined the expression of de2f1-regulated genes in de2f2 and de2f1; de2f2-mutant animals. Third instar eye discs were chosen for this analysis to avoid the possible contribution of maternally supplied products. Maternally supplied dE2F1 is rapidly depleted and zygotic de2f1 expression becomes limiting during embryogenesis. Although dE2F2 is abundant in embryo extracts, dE2F2 protein and mRNA was undetectable in Western and Northern blot analyses of de2f2 or de2f1; de2f2 mutant larvae collected early in the second instar (Fig. 4A; data not shown).

Initially we monitored expression of PCNA, one of the best-characterized E2F-regulated genes, by in situ hy-

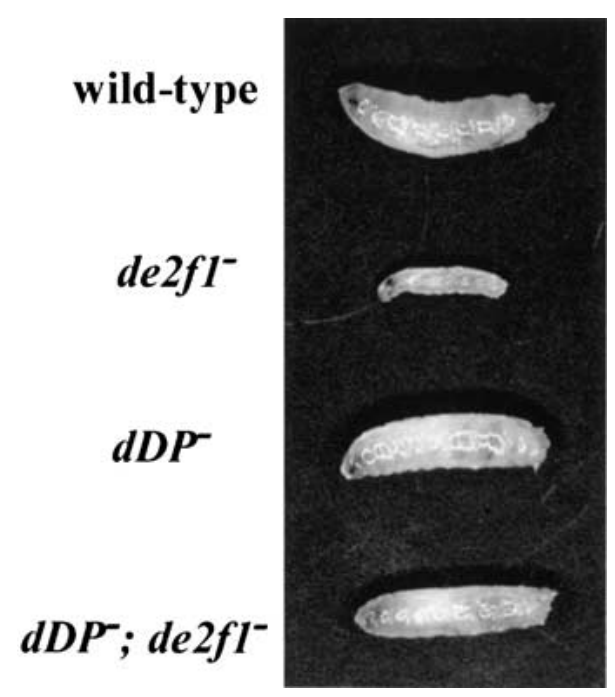

Figure 6. Loss of $d D P$ function rescues the developmental defects of $d e 2 f 1$ mutant larvae. Larvae are shown on the fifth day after egg laying for the following genotypes: Canton $S$ (wildtype), de2f1 $1^{91} / d e 2 f 1^{\text {rm729 }}\left(\right.$ de2f $\left.1^{-}\right), d D P^{a 2} / D f(2 R)_{V g}-B\left(d D P^{-}\right)$, and $d D P^{a 2} / D f(2 R)_{v g}-B$; de2f1 $1^{91} / d e 2 f 1^{r m 729}\left(d D P^{-}\right.$; de2f1-). bridization as described in previous reports. In wild-type eye discs, the pattern of PCNA expression is tightly coupled to the pattern of DNA replication. High levels of PCNA transcripts are present anterior to the furrow and within a narrow stripe that overlaps with the second mitotic wave. No PCNA expression is present in the furrow and in the cells just anterior to the furrow (Fig. 7A). In de2f1; de2f2 double-mutant eye discs, PCNA expression was abnormal. In these animals, a weak staining was observed in mutant eye discs without any specific pattern (Fig. 7B). PCNA transcripts appeared to be present at a low level in the anterior portion of the disc, including the morphogenetic furrow and the second mitotic wave. In de2f2 mutant animals, PCNA expression was not downregulated as cells enter the furrow, and this resulted in an expanded region of PCNA transcription that encompassed both the first and second mitotic waves and the morphogenetic furrow (Fig. 7C).

Although in situ hybridization is an excellent method for monitoring expression patterns, without a suitable internal control it is a poor way for comparing expression levels. To compare the overall levels of E2F-target genes expression, we performed Northern blot analysis using total RNA from third instar eye imaginal discs dissected from de2f2 single and de2f1; de2f2 double-mutant larvae. As shown in Figure 7G, the steady state level of PCNA transcripts was elevated in the de2f2 mutant and declined in the double mutant to a level that was lower than the wild-type control. These results suggest that $\mathrm{dE} 2 \mathrm{~F} 2$ represses PCNA expression and that the elevated level of PCNA expression in the de2f2 mutant is due to the activity of $\mathrm{dE} 2 \mathrm{~F} 1$. The expression of $M C M 3$, another proposed target of dE2F1 (Duronio et al. 1998), increased in the de2f2 single mutant and this effect was suppressed in the double mutant, but in this case the total amount of MCM3 RNA in the double-mutant discs was indistinguishable from wild type. Even though the pattern of RNR2 expression has been shown to be dependent on dE2F1 (Duronio et al. 1995), we saw little change in the total level of RNR2 transcripts in de2f1 mutant larvae (data not shown) or in de2f2, or de2f1; de2f2 mutant eye discs (Fig. 7G).

We conclude that both de2f1 and de2f2 are required for the normal pattern of PCNA expression. de2f2 is needed to repress PCNA expression in the morphogenetic furrow; whereas de2f1 is required for the high levels of expression in the first and second mitotic waves. Although the pattern of PCNA, MCM3, and RNR2 transcripts depends on $d e 2 f 1$ and/or de2f2, significant levels of each of these transcripts can be detected in the absence of both de $2 f 1$ and $d e 2 f 2$.

Because Cyclin E is one of the best-known targets of $\mathrm{E} 2 \mathrm{~F}$ and is rate limiting for S-phase entry we examined the pattern of Cyclin E transcription in the de2f2; de2f1 double-mutant animals. The normal pattern of Cyclin $E$ expression was not altered in de2f2 mutant discs and a similar pattern was also evident in de2f2; de2f1 double mutants. However in the absence of both dE2F1 and $\mathrm{dE} 2 \mathrm{~F} 2$, the variations in Cyclin $E$ expression were reduced and the pattern of expression was less distinct (Fig. 
Frolov et al.

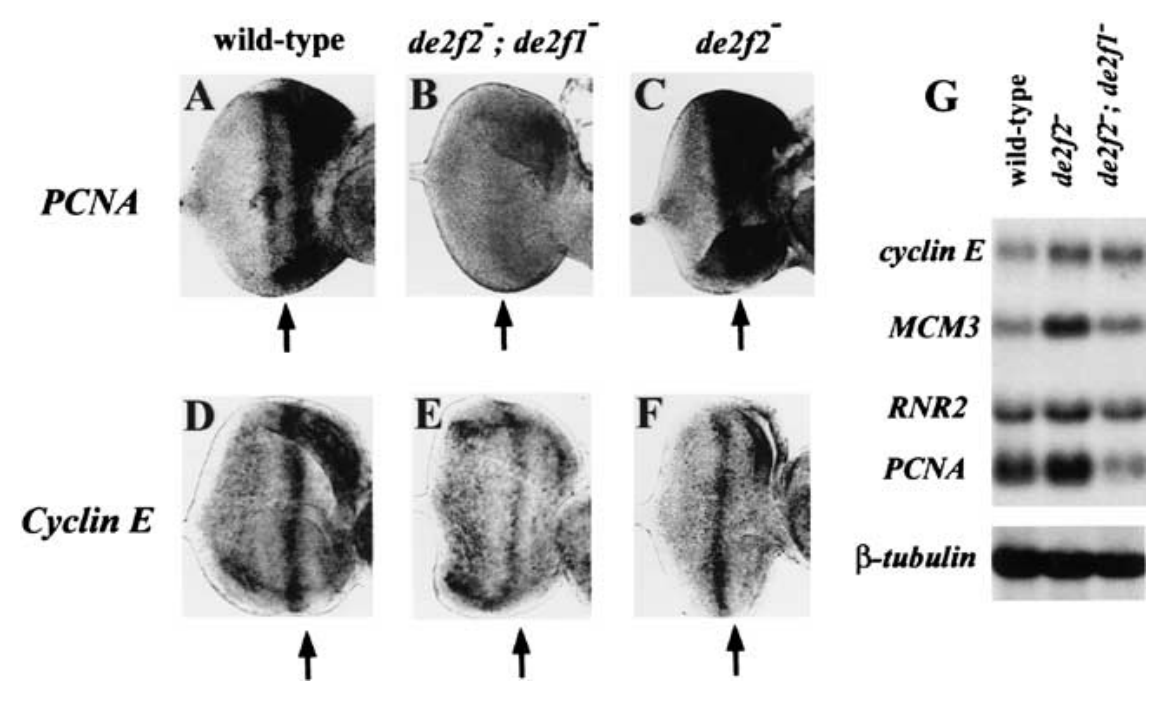

Figure 7. Changes in the expression of E2F-regulated genes in animals lacking de2f1 and de2f2. Data is shown with material from wild type (Canton $S$ ), de2f2 ${ }^{76 Q 1} /$ de $2 f 2^{G 5.1} ; \quad \operatorname{de} 2 f 1^{91} /$ de $2 f 1^{\text {rm729 }}$ and de2f2 $2^{7621} / d e 2 f 2^{G 5.1}$ animals. $(A-F)$ In situ hybridization of the third instar eye discs with probes to PCNA $(A-C)$ or Cyclin $E(D-F)$. Note that PCNA expression is not downregulated anterior to, or within, the morphogenetic furrow (arrow) in de2f2 deficient discs $(C)$ and that PCNA is expressed at low levels without any distinguishable pattern in the absence of both dE2F1 and dE2F2 (B). The pattern of $\mathrm{Cyc}$ lin $E$ expression is normal in de2f2 deficient discs $(F)$ and is also detectable in double-mutant discs, although the variations in Cyclin E expression are less dramatic in the absence of both dE2F1 and dE2F2 (E). (G) Northern blot analysis using total RNA from dissected eye discs, and probes to several E2F regulated genes. Note that the levels of Cyclin E, MCM3, and RNR2 transcripts are similar in wild-type and double-mutant larvae; whereas the levels of PCNA are reduced in the absence of both dE2F1 and dE2F2.

7E). Northern analysis showed that the steady-state level of Cyclin E transcripts was not decreased in the absence of E2F proteins (Fig. 7G). These results are consistent with evidence that de2f1 contributes to the pattern of $C y c l i n$ E expression but is not required for Cyclin E transcription (Duronio and O'Farrell 1995). The finding that E2F target genes are expressed in de2f2; de2f1 double mutants may explain, at least in part, why normal cell proliferation is possible in the absence of E2F proteins.

\section{Discussion}

Drosophila contains only two E2F family members and the results described above show that their gene products have opposite activities and antagonistic effects on cell proliferation during the larval stages of development. We find that dE2F1 and dE2F2 both heterodimerize with $\mathrm{dDP}$, bind to E2F sequences, and interact with RBF. However dE2F1 is a potent activator of transcription, and the loss of de2f1 results in the loss of expression of E2F-regulated genes (Dynlacht et al. 1994; Duronio et al. 1995; Royzman et al. 1997). Conversely, the overexpression of dE2F2 repressed transcription of E2F reporters in transfection experiments and the loss of de2f2 function resulted in increased and expanded patterns of gene expression in vivo. The mechanism of dE2F2-mediated repression is not known. Given that dE2F2 and RBF physically interact and both proteins are required for the repression of the PCNA promoter it seems likely that dE2F2 acts in conjunction with a pocket protein.

The most definitive evidence that $\mathrm{dE} 2 \mathrm{~F} 1$ and $\mathrm{dE} 2 \mathrm{~F} 2$ have opposing functions is the finding that the loss of de2f2 suppresses the proliferation defects in de2f1 mutant larvae. This suppression leads to the surprising conclusion that the strong phenotypes seen in de2f1 mu- tants are not simply due to the absence of dE2F1 but also to the unchecked activity of dE2F2. In the absence of both proteins, larval cell proliferation is relatively normal. This result explains why $d D P$ mutants have a much milder phenotype than de2f1 mutants (Royzman et al. 1997). Based on this set of observations, one could argue that the primary function of dE2F1 at this stage of development is to relieve the effects of dE2F2.

Chromatin immunoprecipitation experiments show that dE2F2 is normally present at promoters that are induced by dE2F1. Thus, the simplest interpretation of this data is that the antagonistic functions of dE2F1 and $\mathrm{dE} 2 \mathrm{~F} 2$, result from their opposing effects on the same set of target genes (Fig. 8). In this model, we suggest that E2F-mediated repression limits the rate of cell proliferation in the imaginal disc, and that the burst of E2F-regulated gene expression that precedes and parallels S-phase entry results from the ability of dE2F1-mediated activation to override dE2F2-mediated repression. This idea fits with the observation that relatively low levels of dE2F1 are able to overcome much higher levels of dE2F2 in transient transfection experiments and in transgenic animals, and with the patterns of dE2F1 and dE2F2 expression in the eye disc. Whereas dE2F2 appears to be expressed throughout the eye disc, high levels dE2F1 are restricted to areas containing asynchronously dividing cells, and are seen transiently in the second mitotic wave (Asano et al. 1996; Brook et al. 1996). The tight regulation of dE2F1 expression, and the idea that dE2F1 normally overrides dE2F2, may explain why the loss of dE2F2 does not give rise to widespread inappropriate proliferation.

In the absence of both dE2F1 and dE2F2, gene expression is presumably dependent on other transcription factors that bind to these promoters and the outcome is expected to vary depending on the factors that are pres- 


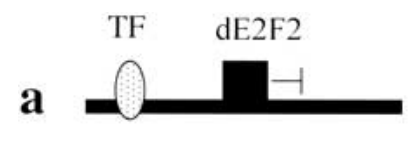

$\mathrm{dE} 2 \mathrm{~F} 2$ represses gene expression

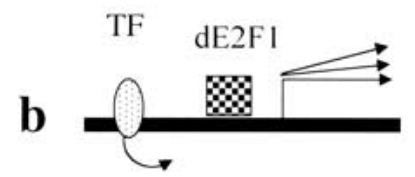

$\mathrm{dE} 2 \mathrm{~F} 1$ overrides $\mathrm{dE} 2 \mathrm{~F} 2$ allowing a burst of gene expression.

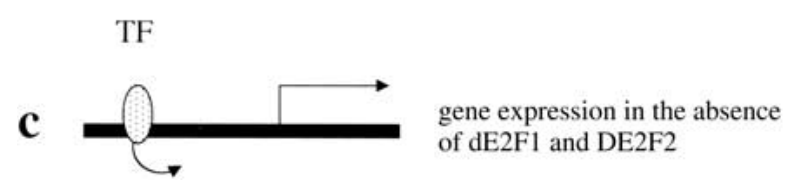

Figure 8. Model for the regulation of E2F-dependent transcription during larval development. Two functionally different dE2F complexes can occupy E2F-regulated promoters. One complex contains dE2F2 and represses transcription, most likely via interaction with RBF and RBF-associated proteins $(A)$. As dE2F2 is ubiquitously expressed at high levels, we suggest that it is present on most vacant promoters. During the $G_{1} / S$ transition, newly synthesized dE2F1 binds to the target promoters and overrides the activity of dE2F2 $(B)$, allowing a burst of gene expression as cells enters S-phase. The de2f1 mutant phenotype is caused by the failure of dE2F1 to override dE2F2 and the unchecked activity of dE2F2. In double-mutant animals $(C)$ the loss of dE2F1-mediated activation is compensated by the loss of dE2F2-mediated repression, and the expression of target genes is regulated by other transcription factors $(\mathrm{TF})$ that bind to these promoters.

ent and the net contribution of the E2F-binding sites to activation or repression. We note that several factors have been found to bind to the PCNA promoter, in addition to E2F (Yamaguchi et al. 1996; Hayashi et al. 1997; Sawado et al. 1998a; Hayashi et al. 1999), and that mutation of all three E2F-binding sites in DNApola promoter reduces, but does not abolish, transcription in transgenic animals (Yamaguchi et al. 1997). Moreover most studies of E2F-regulated promoters in mammalian cells show that the mutation of E2F-binding sites alters the timing of gene expression but does not block transcription (Means et al. 1992; Johnson et al. 1994). Clearly, the level of E2F-independent transcription is sufficient for relatively normal rates of growth and cell proliferation within the imaginal discs.

Precisely how dE2F1 normally overrides dE2F2 is unclear. It is possible that dE2F1 simply replaces dE2F2 on key regulatory sites. However many E2F-regulated promoters contain multiple E2F-binding sites and it is possible that $\mathrm{dE} 2 \mathrm{~F} 1$ and $\mathrm{dE} 2 \mathrm{~F} 2$ act through distinct sites on the same set of promoters. To date, no experiments have unequivocally identified all of the transcriptional targets of E2F that are most important for cell proliferation. Thus it is possible that, although dE2F1 and dE2F2 have opposite effects on the expression of some common targets, they exert their antagonistic effects on cell proliferation by regulating different subsets of target genes. It is also possible that $\mathrm{dE} 2 \mathrm{~F} 2$ has a gain-of-function effect in the absence of dE2F1, and blocks the expression of a subset of genes that it would never normally regulate. Finally, it is conceivable that either dE2F1 or dE2F2, or both, act directly at origins of replication (Royzman et al. 1999). However, that the relatively normal patterns of BrdU incorporation in de2f1; de2f2 mutant larvae indicates that neither of these proteins have an essential function in DNA replication at this stage of development.

The rescue of cell proliferation defects in de2f1 mutant larvae by mutation of de2f2 does not imply that E2F-regulation is nonessential in Drosophila. de2f1; de2f2 double mutants die during pupation and we have not yet observed any adult escapers that completely lack both dE2F1 and dE2F2. At present it is uncertain whether the E2F function needed during the pupal stage is unique to de2f1 or is redundant between de2f1 and de2f2. Conceivably, the pupal lethality of de2f1; de2f2 mutants could result from a defect in either the differentiation, or proliferation, or survival of a critical cell type. Because $\mathrm{dE} 2 \mathrm{~F} 1$ and $\mathrm{dE} 2 \mathrm{~F} 2$ both form complexes with RBF in vivo, it is feasible that both proteins may mediate transcriptional repression in some cell types. It is also evident that the individual dE2F proteins do have unique functions that are essential at specific stages of Drosophila development. For example, the work of Cayirlioglu and coworkers suggests that dE2F2 has a unique role in the shut-off of DNA endoreplication in follicle cells (Cayirlioglu et al., in prep.), and Orr-Weaver and colleagues have shown that dE2F1 is required for the control of chorion gene amplification in this same cell type (Royzman et al. 1999). Thus the functional antagonism apparent between $d e 2 f 1$ and $d e 2 f 2$ may be a characteristic of proliferating cells and may not apply to all cell types and all stages of development.

Thus far, studies of the E2F/RB pathway in Drosophila have given results that closely parallel studies in mammalian cells. Although E2F-1, E2F-2, E2F-3, E2F-4, and E2F-5 are all able to activate transcription and be repressed by pRB-family members, E2F-1, E2F-2, and E2F-3 are generally more tightly linked to activation of transcription; whereas E2F-4 and E2F-5 are the family members most strongly linked to repression. We speculate that the function of $\mathrm{dE} 2 \mathrm{~F} 1$ may resemble the role played by the strong activator E2F;s, while the role of dE2F2 may resemble the functions of the corepressor E2Fs. Intriguingly, E2f-3-deficient MEFs show a proliferation defect that resembles the de2f1 mutant phenotype; conversely E2f-4; E2f-5 double-mutant MEFs have defects in cell cycle arrest (Bruce et al. 2000; Gaubatz et al. 2000). By analogy with the Drosophila E2Fs, it will be interesting to determine whether any defects in cell proliferation caused by the mutation of E2F-3, E2F-2, or E2F-1 (either singly or in combination) can be suppressed by inactivation of E2F-4 or E2F-5.

These results highlight two general issues. One implication of this work concerns the role of E2F in cell cycle control. Many studies have shown that E2F proteins are potent regulators of the cell cycle; either increasing or decreasing E2F activity can have striking effects on cell cycle progression. However, we find that fairly normal 
patterns of cell proliferation are possible in the absence of both de2f1 and de2f2. This indicates that, while the activities of individual E2F proteins do affect cell cycle progression, E2F-regulation is not an obligate component of the cell cycle machinery.

Second, this study shows one of the many complications that can arise when one member of a family of related proteins is specifically removed. In the case of E2F, several different family members can target the same promoter elements. Thus, the phenotype that is seen when one E2F family member is removed reflects a combination of changes that involves both the absence of the targeted gene product and the altered importance of the E2F complexes that remain in the cell. To some extent, the effect of removing activator E2Fs could be regarded as an increase in the activity of repressor E2Fs or vice-versa. In normal cells, E2F-mediated control of cell proliferation results from the balanced interplay between E2Fs with different, even opposing, activities.

\section{Materials and methods}

\section{Generation of the de2f2 mutant}

de2f2 maps to the base of the left arm of chromosome 2 at position 39B2-3. A semilethal $P$ element line, 1(2)k16403, containing an insertion 667 bp upstream of the $5^{\prime}$ end of de2f2 was used to generate mutant alleles of de2f2. The insertion is responsible for the lethality, because excision of the $P$ element reverts the lethal phenotype.

Analysis of the genomic sequence revealed a putative ORF in close proximity of the $P$-element insertion in $1(2) \mathrm{k} 16403$. A corresponding cDNA was cloned using a Rapid Amplification of cDNA Ends (RACE) protocol and the transcription map of this region was established (Fig. 9). The $P$ element in $1(2)$ k16403 is
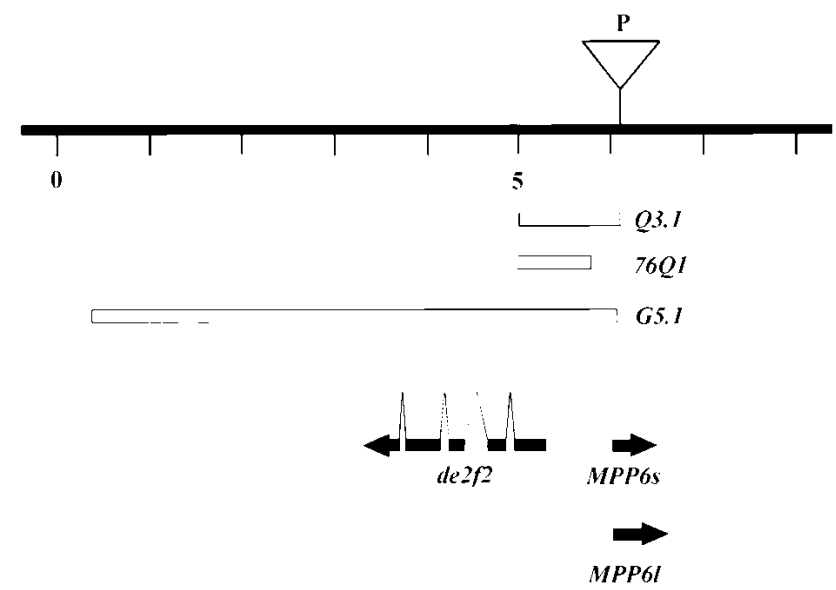

Figure 9. Molecular map of the de2f2 region. Position of the $P$-element insertion in $1(2) \mathrm{k} 16403$ is designated by a triangle. de2f2 alleles de2f2Q3.1 (Q3.1) and de2f2G5.1 (G5.1) were generated in the first round of male recombination; de2f2 ${ }^{76 Q 1}$ (76Q.1) was made in the second round of male recombination from the de2f2 ${ }^{\mathrm{Q} .1}$ chromosome. Open boxes show sequences deleted in the respective alleles. Exon-intron structure of transcripts synthesized in the region. MPP6 unit produces two mRNAs (MPP6s and MPP61) with alternative polyadenylation sites. inserted in the $5^{\prime}$ UTR of the MPP6 gene, 16 bp downstream of the transcription start site (GenBank accession no. AY029612). MPP6 encodes a Drosophila homolog of a human M-phase phosphoprotein 6 (Matsumoto-Taniura et al. 1996).

A standard approach to make a null allele by using a preexisting $P$-element insertion is to generate imprecise excisions of the $P$ element. Deletions generated in this way from $1(2)$ k16403 would automatically remove MPP6 sequences. To overcome this problem we employed a different approach based on the phenomenon of male recombination. It is known that mobilization of $P$ element results in significant increase in frequency of crossing over in the male germline, with the breakpoint being within several kilobases of an existing $P$ element. Moreover, most of the recombinants carry either a deletion or a duplication of the genomic sequences immediately flanking the $P$-element insertion site (Preston et al. 1996). The major advantage of this approach is that the generated aberrations retain a mobile $P$ element. Hence, this chromosome could be subsequently used in following rounds of recombination. The recombinants can be identified with the distant flanking chromosomal markers. The strategy for the generation of the de2f2 mutant is briefly outlined below. In the first round, rearranged chromosomes carrying deletions extending distally from the $P$-element insertion into de2f2 were selected. In the next step, these deletions were used to generate small duplications to restore the sequences of MPP6 but not de2f2.

For the first round of male recombination, jump-start males 1(2) k16403/dp b cn bw; $\Delta 2-3 \mathrm{Dr} /+$ were crossed to $d p b \mathrm{cn} b w$ females. Potential recombination events in $1(2) \mathrm{k} 16403 / d p b \mathrm{cn}$ $b w ; \Delta 2-3 D r /+$ males were revealed by segregation among the second-chromosome distal markers and found to be at frequency $\sim 0.2 \%$. Males that are $d p b c n^{+} b w^{+}$presumably carry either a distal deletion or proximal duplication. A total of 18 males were crossed individually to $S p / C y O, d p c n$ bw to establish stocks. As revealed by PCR analysis, in two stocks, de2f2 ${ }^{G 5.1}$ and $d e 2 f 2^{Q 3.1}$, the recombination event resulted in a distal deletion that removed de2f2; their precise position was determined by sequencing. In de2f2 ${ }^{Q 3.1}$ allele $870 \mathrm{bp}$ distal to the $P$-element insertion site are removed, while in $d e 2 f 2^{G 5.1}$ the deletion extends $5621 \mathrm{bp}$.

To restore the integrity of the MPP6 promoter, both alleles were used for a second round of recombination. First, $d p^{+}$and $b^{+}$ markers were recombined on the de2f2 ${ }^{\mathrm{Q} 3.1}$ and $d e 2 f 2^{G 5.1}$ chromosomes. Then, jump-start males, de2f2 $2^{\mathrm{Q} 3.1} / \mathrm{dp} b \mathrm{cn} b w ; \Delta 2-3$ $D r /+$ or $d e 2 f 2^{G 5.1} / d p b c n b w ; \Delta 2-3 D r /+$, were crossed to $d p b c n$ $b_{w}$ females. Seventy-five $d p^{+} b^{+} c n b w$ recombinants were isolated and analyzed by PCR and sequencing. In 53 cases, recombination was accompanied by the precise excision of the $P$-element and 12 chromosomes were identical to parental; in three cases proximal deletions were generated and in four cases the structure was not determined. Three chromosomes carried distal duplications and two of them originated from the $\operatorname{de} 2 f 2^{G 5.1}$ chromosome were large and restore de2f2. In one case a duplication event from the de2f2 ${ }^{\mathrm{Q} 3.1}$ chromosome was recovered. In the generated duplication, de2f2 ${ }^{76 \mathrm{Q} 1}, 273 \mathrm{bp}$ upstream of MPP6 were restored.

\section{Fly stocks}

The following alleles were used: $d e 2 f 1^{91}$ and $d e 2 f 1^{r m 729}$ are null alleles for de2f1 (Duronio et al. 1995), $d D P^{a 2}$ is a null allele of $d D P$ (Royzman et al. 1997), $D f(2 R) v g-B$ is a deficiency uncovering the $d D P$ gene (Royzman et al. 1997), MPP6 U39 is a point mutation in $M P P 6$, which converts the sixth amino acid into a stop codon and will be described elsewhere (D.S. Huen, unpubl.). 
To analyze various mutant combinations, the mutant alleles were balanced over $T(2 ; 3) C y O T M 6 B, C y T b$ translocation. All mutations were analyzed in trans-heterozygous conditions. Trans-heterozygous mutant larvae were identified by the absence of a dominant marker Tb. Trans-heterozygous de2f2 mutant larvae were collected from the cross of de2f276Q1/ T(2;3)CyO TM6B, Cy Tb and de2f2 ${ }^{G 5.1} / T(2 ; 3)$ CyO TM6B, Cy $T b$. de2f1; de2f2 double mutants were collected by crossing de2f2 ${ }^{76 \mathrm{Q} 1}$; de2f1 ${ }^{\text {rm729 }} / \mathrm{T}(2 ; 3) \mathrm{CyO} T \mathrm{TM} 6 \mathrm{~B}, \mathrm{Cy} \mathrm{Tb}$ to de2f2 ${ }^{\mathrm{G5} .1}$; de2f191/T(2;3)CyO TM6B, Cy $T b$. To make de2f1; $d D P$ null larvae, $d D P^{a 2}$; de2 $f 1^{91} / T(2 ; 3) C y O T M 6 B, C y T b$ flies were crossed to $D f(2 R)_{v g}-B$; de2f1 ${ }^{r m 729} / T(2 ; 3) C y O T M 6 B, C y T b$.

For $P$-element transformation, the de2f2 cDNA was cloned into the pUAST vector and injected with the $\Delta 2-3$ helper plasmid into $y w^{1118}$ embryos. Several independent lines on each chromosome were established.

\section{DNA and RNA manipulations}

All standard DNA manipulations were performed as described (Sambrook et al. 1989). Total RNA was isolated from third instar eye discs using Trizol (GIBCO BRL). Approximately $5 \mu \mathrm{g}$ of RNA isolated from 50 discs were loaded per lane. The RNA was fractionated on $1 \%$ agarose gels containing $2.2 \mathrm{M}$ formaldehyde. The RNA was transferred by capillary blotting in 10× SSC from the gel to neutral nylon membranes (S\&S) and then hybridized to radio-labeled antisense RNA probes prepared by in vitro transcription with T3 or T7 RNA polymerase (Promega). The prehybridization and hybridization solutions were $0.5 \mathrm{M} \mathrm{NaCl}, 7 \%$ dextran sulfate, $0.2 \mathrm{mg} / \mathrm{mL}$ heparin, $1 \%$ sarkosyl, $0.1 \mathrm{M}$ sodium phosphate $(\mathrm{pH} 7.0), 0.1 \%$ ficoll, $0.1 \%$ polyvinylpyrrolidone, $0.1 \%$ sodium pyrophosphate, and $50 \%(\mathrm{v} / \mathrm{v})$ formamide. The filters were washed three times in a solution, containing $5 \mathrm{mM}$ sodium phosphate, $0.2 \%$ SDS, and $1 \mathrm{mM}$ EDTA for $1 \mathrm{~h}$ at $75^{\circ} \mathrm{C}$.

To obtain the $5^{\prime}$ and $3^{\prime}$ ends of the transcripts synthesized with the de2f2 region, the RACE protocol was performed using the Marathon cDNA Amplification Kit (Clontech) according to the manufacturer's recommendations. As a template for cDNA synthesis, the poly(A)+ RNA was isolated from Canton S adult flies using a polyAT track kit (Promega).

In situ hybridization with the eye discs was performed as described (Du 2000). PCNA and Cyclin E riboprobe were prepared by in vitro transcription with DIG RNA labeling mix and detected with alkaline phosphatase conjugated anti-DIG antibody (Boehringer-Mannheim).

\section{Antibodies, Western blots, and immunoprecipitations}

Mice were immunized with $\mathrm{dE} 2 \mathrm{~F} 2$ protein expressed in Escherichia coli. The dE2F2 monoclonal antibodies mei3 and mei8 were prepared by fusing splenocytes to NS-1 myeloma cells as described (Harlow and Lane 1988). mei8 was found to be specific for dE2F2 in Western blotting; however, mei3 was specific in immunoprecipitation experiments. A rabbit antiserum to dE2F2 was raised by injecting a rabbit with the recombinant full-length dE2F2 protein. dE2F2 polyclonal antibodies were affinity purified over a GST-dE2F2 column.

Mouse monoclonal anti-dDP (Yun mix) and mouse monoclonal anti-RBF (DX-3) were described previously (Du et al. 1996a,b). IP-Westerns on SL2 cells were performed by standard methods (Du et al. 1996a). The interactions between dE2F2, $\mathrm{dDP}$, and RBF were not disrupted by EtBr treatment. For immunoprecipitations, anti-dE2F2 mei3 antibodies were used at 1:1 and anti-dDP at 1:1 dilution. For Western blot analysis, antidE2F2 mei8 antibody were used at 1:2, anti-RBF at 1:2 and anti$\mathrm{dDP}$ at 1:3. Peroxidase-conjugated anti-rabbit and anti-mouse secondary antibodies were from Amersham. For Western blot analysis of trans-heterozygous de2f2 mutant animals, non- $T b$ second instar larva were collected from the cross between de2f2 $2^{76 Q 1} / T(2 ; 3) C y O T M 6 B, C y ~ T b$ and $d e 2 f 2^{G 5.1} / T(2 ; 3) C y O$ $T M 6 B, C y T b$ and the extracts were prepared following the protocol for SL2 cells.

\section{Transient transfections}

For transfections, the dE2F1 and dE2F2 ORFs were cloned into pIE4 vector (Novagen) under the control of baculovirus ie1 promoter and the constructs were verified by sequencing. Drosophila SL2 cells were transfected with CellFectin (GIBCO BRL) with $10 \mu \mathrm{g}$ of plasmid DNA. The reporter constructs consists of the luciferase gene fused to either the wild-type PCNA promoter, $5^{\prime}$-168DPCNAluc or the PCNA promoter with mutated E2F sites, p5'-168DPCNAmutI\&mutIIluc (Sawado et al. 1998b). To determine the efficiency of transfection and to normalize the luciferase read-outs, the $\beta$-galactosidase expressing construct, pIE4-lacZ, was included in each experiment. Cells were harvested $36 \mathrm{~h}$ after transfection and assayed for $\beta$-galactosidase and luciferase activity by standard methods (Dynlacht et al. 1994).

\section{Chromatin immunoprecipitations}

Chromatin immunoprecipitations were performed using modified methods published previously (Dimova et al. 1999; Meluh and Broach 1999). Drosophila SL2 cells were grown at $25^{\circ} \mathrm{C}$ in spinner flasks in Schneider's Drosophila medium (GIBCO BRL) supplemented with $10 \%$ fetal calf serum. Formaldehyde was added to the tissue culture medium to a final concentration of $1 \%$ and cross-linking was performed for $15 \mathrm{~min}$ at room temperature and terminated with glycine (final concentration 0.125 M). The equivalent of $\sim 10^{8}$ cells were used per immunoprecipitation reaction and incubated with either $2 \mu \mathrm{L}$ of anti-dE2F2 polyclonal rabbit antibody, with $2 \mu \mathrm{L}$ of anti-dE2F1 polyclonal guinea pig antibody, with rabbit anti-mouse whole serum (nonspecific antibodyl, or without an antibody. Analysis of DNA was performed using multiplex PCR. The following primer sets were used: PCNA, 5'-GCAGAGCTGCGTGGAATGATTAAA GC-3' and 5'-TTGACCCAGGCGTGCCTCGAACATAT-3'; DNA pol $\alpha$, 5'-CAGGTCGGATTTCCCGCCAAAATA-3' and 5'-GTGACCAGGGATGGAGGATGATCA-3'; rp49, 5' -TCCG GCAAGGTATGTGCGTGATTT-3' and 5'-ATATCGATCCG ACTGGTGGCGGAT-3'. PCR products were resolved on $6 \%$ polyacrylamide gels.

\section{Immunofluorescence}

For immunostaining, rabbit affinity purified anti-dE2F2 antibodies were used at a 1:100 dilution, anti-Cyclin A (Knoblich and Lehner 1993) at 1:100, anti-phospho-Histone $\mathrm{H} 3$ (Upstate Biotechnology) at 1:200, and rat anti-elav (O'Neill et al. 1994) at 1:200. For BrdU labeling experiments, eye discs from third instar larvae were dissected in Schneider's medium for $10 \mathrm{~min}$ and labeled with $0.2 \mathrm{mg} / \mathrm{mL} \mathrm{BrdU}$ (Sigma) at room temperature. Eye discs were fixed and BrdU was detected with mouse anti-BrdU antibody (Becton Dickinson) at 1:50. Secondary antibodies were Cy5 donkey anti-rabbit, Cy3 donkey anti-mouse (Jackson Immunolaboratories), and Alexa Fluor 488 anti-rat (Molecular Probes). Images were collected on a Zeiss LSM510 confocal microscope and processed with Adobe Photoshop 5.5.

\section{Acknowledgments}

We thank Adelaide Carpenter, John Roote, and Yian-Rong Chen for expert technical assistance, Bloomington Drosophila Stock 
Center for the provision of stocks, and our colleagues for valuable discussions. We thank Dr. Terry Orr-Weaver for the kind gift of dE2F1 antisera; Drs. Masamitsu Yamaguchi and Peter Bangs for the reporter constructs; Dr. Bob Duronio for exchanging information prior to publication; Dr. Nick Tapon for help with confocal microscopy; and Drs. Iswar Hariharan, Ken Moberg, and Ed Harlow for insightful comments on the manuscript. D.D. was supported by an NIH training grant. D.S.H. was supported by grants from the Cancer Research Campaign and Medical Research Council. The provision of laboratory facilities and technical support by B.A.J. Ponder and M. Ashburner is gratefully acknowledged by D.S.H. This work was supported by NIH grant number GM53203 (N.D.), a CRC program grant and an MRC program grant to M. Ashburner, S. Russell, and D. Gubb.

The publication costs of this article were defrayed in part by payment of page charges. This article must therefore be hereby marked "advertisement" in accordance with 18 USC section 1734 solely to indicate this fact.

\section{References}

Adams, M.R., Sears, R., Nuckolls, F., Leone, G., and Nevins, J.R. 2000. Complex transcriptional regulatory mechanisms control expression of the E2F3 locus. Mol. Cell. Biol. 20: 36333639.

Aravind, L., Dixit,V.M., and Koonin, E.V. 2001. Apoptotic molecular machinery: Vastly increased complexity in vertebrates revealed by genome comparisons. Science 291: 12791284.

Asano, M., Nevins, J.R., and Wharton, R.P. 1996. Ectopic E2F expression induces $\mathrm{S}$ phase and apoptosis in Drosophila imaginal discs. Genes \& Dev. 10: 1422-1432.

Brehm, A., Miska, E.A., McCance, D.J., Reid, J.L., Bannister, A.J., and Kouzarides, T.E. 1998. Retinoblastoma protein recruits histone deacetylase to repress transcription. Nature 391: 597-601.

Brook, A., Xie, J.-E., Du, W., and Dyson, N. 1996. Requirements for $\mathrm{dE} 2 \mathrm{~F}$ function in proliferating cells and in post-mitotic differentiating cells. EMBO J. 15: 3676-3683.

Bruce, J.L., Hurford, R.K., Classon, M., Koh, J., and Dyson, N. 2000. Requirements for cell cycle arrest by p16INK4a. Mol. Cell 6: 737-742.

de Nooij, J.C., Letendre, M.A., and Hariharan, I.K. 1996. A cyclin-dependent kinase inhibitor, dacapo, is necessary for timely exit from the cell cycle during drosophila embryogenesis. Cell 87: 1237-1247.

DeGregori, J., Leone, G., Miron, A., Jakoi, L., and Nevins, J.R. 1997. Distinct roles for E2F proteins in cell growth control and apoptosis. Proc. Natl. Acad. Sci. 94: 7245-7250.

Dimova, D., Nackerdien, Z., Furgeson, S., Eguchi,S., and Osley, M.A. 1999. A role for transcriptional repressors in targeting the yeast Swi/Snf complex. Mol. Cell 4: 75-83.

Dobrowolski, S.F., Stacey, D.W., Harter, M.L., Stine, J.T., and Hiebert, S.W. 1994. An E2F dominant negative mutant blocks E1A induced cell cycle progression. Oncogene 9: 2605-2612.

$\mathrm{Du}, \mathrm{W}$. 2000. Suppression of the $r b f$ null mutants by a de2f1 allele that lacks transactivation domain. Development 127: 367-379.

Du, W., Vidal, M., Xie, J.-E., and Dyson, N. 1996a. RBF, a novel RB-related gene that regulates E2F activity and interacts with cyclin E in Drosophila. Genes \& Dev. 10: 1206-1218.

Du, W., Xie, J.-E., and Dyson, N. 1996b. Ectopic expression of $\mathrm{dE} 2 \mathrm{~F}$ and $\mathrm{dDP}$ induces cell proliferation and death in the
Drosophila eye. EMBO J. 15: 3684-3692.

Duronio, R.J. and O'Farrell, P.H. 1995. Developmental control of the $\mathrm{G}_{1}$ to $\mathrm{S}$ transition in Drosophila; cyclin $\mathrm{E}$ is a limiting downstream target of E2F. Genes \& Dev. 9: 1456-1468.

Duronio, R.J., O'Farrell, P.H., Xie, J.-E., Brook, A., and Dyson, N. 1995. The transcription factor E2F is required for S phase during Drosophila embryogenesis. Genes \& Dev. 9: 14451455.

Duronio, R.J., Bonnette, P.C., and O'Farrell, P.H. 1998. Mutations of the Drosophila dDP, dE2F, and cyclin E genes reveal distinct roles for the E2F-DP transcription factor and cyclin E during the S-phase transition. Mol. Cell. Biol. 18: 141-151.

Dynlacht, B.D., Brook, A., Dembski, M.S., Yenush, L., and Dyson, N. 1994. DNA-binding and trans-activation properties of Drosophila E2F and DP proteins. Proc. Natl. Acad. Sci. 91: 6359-6363.

Dyson, N. 1998. The regulation of E2F by pRB-family proteins. Genes \& Dev. 12: 2245-2262.

Flemington, E.K., Speck, S.H., and Kaelin, Jr., W.G. 1993. E2F-1 mediated transactivation is inhibited by complex formation with the retinoblastoma susceptibility gene product. Proc. Natl. Acad. Sci. 90: 6914-6918.

Fry, C.J., Slansky, J.E., and Farnham, P.J. 1997. Position-dependent transcriptional regulation of the murine dihydrofolate reductase promoter by the E2F transactivation domain. Mol. Cell. Biol. 17: 1966-1976.

Gaubatz, S., Lindeman, G.J., Jakoi, L., Nevins, J.R., Livingston, D.M., and Rempel., R.E. 2000. E2F4 and E2F5 play an essential role in pocket protein-mediated G1 control. Mol. Cell 6: 729-735.

Hao, X.F., Alphey, L., Bandara, L.R., Lam, E.W.-F., Glover, D., and La Thangue, N.B. 1995. Functional conservation of the cell cycle-regulating transcription factor DRTF1/E2F and its pathway of control in Drosophila melanogaster. J. Cell Sci. 108: 2945-2954.

Harbour, J.W. and Dean., D.C. 2000. The Rb/E2F pathway: Expanding roles and emerging paradigms. Genes \& Dev. 14: 2393-2409.

Harlow, E. and Lane, D. 1988. Antibodies: A laboratory manual. Cold Spring Harbor Press, Cold Spring Harbor, NY.

Hayashi, Y., Hirose, F., Nishimoto, Y., Shiraki, M., Yamagishi, M., Matsukage, A., and Yamaguchi, M. 1997. Identification of CFDD (common regulatory factor for DNA replication and DREF genes) and role of its binding site in regulation of the proliferating cell nuclear antigen gene promoter. J. Biol. Chem. 272: 22848-22858.

Hayashi, Y., Yamagishi, M., Nishimoto,Y., Taguchi, O., Matsukage, A., and Yamaguchi, M. 1999. A binding site for the transcription factor Grainyhead/Nuclear transcription factor-1 contributes to regulation of the Drosophila proliferating cell nuclear antigen gene promoter. J. Biol. Chem. 274: 35080-35088.

Helin, K. 1998. Regulation of cell proliferation by the E2F transcription factors. Curr. Opin. Genet. Dev. 8: 28-35.

Helin, K., Harlow, E., and Fattaey, A.R. 1993. Inhibition of E2F-1 transactivation by direct binding of the retinoblastoma protein. Mol. Cell. Biol. 13: 6501-6508.

Hsiao, K.-M., McMahon, S.L., and Farnham, P.J. 1994. Multiple DNA elements are required for the growth regulation of the mouse E2F1 promoter. Genes \& Dev. 8: 1526-1537.

Humbert, P.O., Rogers, C., Ganiatsas, S., Landsberg, R.L., Trimarchi, J.M., Dandapani, S., Brugnara, C., Erdman, S., Schrenzel, M., Bronson, R.T., and Lees, J.A. 2000a. E2F4 is essential for normal erythrocyte maturation and neonatal viability. Mol. Cell 6: 281-291.

Humbert, P.O., Verona, R., Trimarchi, J.M., Rogers, C., Dan- 
dapani, S., and Lees, J.A. 2000b. E2f3 is critical for normal cellular proliferation. Genes \& Dev. 14: 690-703.

Ishizaki, J., Nevins, J.R., and Sullenger, B.A. 1996. Inhibition of cell proliferation by an RNA ligand that selectively blocks E2F function. Nature Med. 2: 1386-1389.

Johnson, D.G., Schwarz, J.K., Cress, W.D., and Nevins, J.R. 1993. Expression of transcription factor E2F1 induces quiescent cells to enter S phase. Nature 365: 349-352.

Johnson, D.G., Ohtani, K., and Nevins, J.R. 1994. Autoregulatory control of E2F-1 expression in response to positive and negative regulators of cell cycle expression. Genes \& Dev. 8: 1514-1525.

Knoblich, J.A. and Lehner, C.F. 1993. Synergistic action of Drosophila cyclins $\mathrm{A}$ and $\mathrm{B}$ during $\mathrm{G} 2 \mathrm{-M}$ transition. EMBO $\mathrm{F}$. 12: $65-74$.

Lam, E.W.-F. and Watson, R.J. 1993. An E2F-binding site mediates cell-cycle regulated repression of mouse B-myb transcription. EMBO J. 12: 2705-2713.

Lam, E.W., Bennett, J.D., and Watson, R.J. 1995. Cell-cycle regulation of human B-myb transcription. Gene 160: 277-281.

Leone, G., Nuckolls, F., Ishida, S., Adams, M., Sears, R., Jakoi, L., Miron, A., and Nevins, J.R. 2000. Identification of a novel E2F3 product suggests a mechanism for determining specificity of repression by Rb proteins. Mol. Cell. Biol. 20: 36263632.

Lindeman, G.J., Dagnino, L., Gaubatz, S., Xu, Y., Bronson, R., Warren, H.B., and Livingston, D.M. 1998. A specific, nonproliferative role for E2F-5 in choroid plexus function revealed by gene targeting. Genes \& Dev. 12: 1092-1098.

Lukas, J., Petersen, B.O., Holm, K., Bartek, J., and Helin, K. 1996. Deregulated expression of E2F family members induces S-phase entry and overcomes p16INK4A-mediated growth suppression. Mol. Cell. Biol. 16: 1047-1057.

Luo, R.X., Postigo, A.A., and Dean, D.C. 1998. Rb interacts with histone deacetylase to repress transcription. Cell 92: 463 473.

Magnaghi-Jaulin, L., Groisman, R., Naguibneva, I., Robin, P., Lorain, S., Le Villain, J.P., Troalen, F., Trouche, D., and Harel-Bellan, A. 1998. Retinoblastoma protein represses transcription by recruiting a histone deacetylase. Nature 391: 601-604.

Matsumoto-Taniura, N., Pirollet, F., Monroe, R., Gerace, L., and Westendorf, J.M. 1996. Identification of novel M phase phosphoproteins by expression cloning. Mol. Biol. Cell 7: 14551469.

Means, A.L., Slansky, J.E., McMahon, S.L., Knuth, M.W., and Farnham, P.J. 1992. The HIP binding site is required for growth regulation of the dihydrofolate reductase promoter. Mol. Cell. Biol. 12: 1054-1063.

Meloni, A.R., Smith, E.J., and Nevins, J.R. 1999. A mechanism for $\mathrm{Rb} / \mathrm{p} 130$-mediated transcription repression involving recruitment of the CtBP corepressor. Proc. Natl. Acad. Sci. 96: 9574-9579.

Meluh, P.B. and Broach, J.R. 1999. Immunological analysis of yeast chromatin. Methods Enzymol. 304: 414-430.

Moberg, K., Starz, M.A., and Lees, J.A. 1996. E2F-4 switches from p130 to p107 and pRB in response to cell cycle reentry. Mol. Cell. Biol. 16: 1436-1449.

Muller, H., Moroni, M., Vigo, E., Peterson, B.O., Bartek, J., and Helin, K.. 1997. Induction of S-phase entry by E2F transcription factors depends on their nuclear localization. Mol. Cell. Biol. 17: 5508-5520.

Muller, H., Bracken, A.P., Vernell, R., Moroni, M.C., Christians, F., Grassilli, E., Prosperini, E., Vigo, E., Oliner, J.D., and K. Helin. 2001. E2Fs regulate the expression of genes involved in differentiation, development, proliferation, and apoptosis.
Genes \& Dev. 15: 267-285.

Myster, D.L., Bonnette, P.C., and Duronio, R.J. 2000. A role for the DP subunit of the E2F transcription factor in axis determination during Drosophila oogenesis. Development 127: 3249-3261.

Neufeld, T.P., de la Cruz, A.F.A., Johnston, L.A., and Edgar, B.A. 1998. Coordination of cell growth and division by Drosophila E2F. Cell 93: 1183-1193.

Neuman, E., Flemington, E.K., Sellers, W.R., and Kaelin, W.G. 1994. Transcription of the E2F1 gene is rendered cell cycledependent by E2F DNA binding sites within its promoter. Mol. Cell. Biol. 14: 6607-6615.

Nevins, J. 1998. Toward an understanding of the functional complexity of the E2F and retinoblastoma families. Cell Growth Differ. 9: 585-593.

Ohtani, K. and Nevins, J.R. 1994. Functional properties of a Drosophila homolog of the E2F1 gene. Mol. Cell. Biol. 14: 1603-1612.

O'Neill, E.M., Rebay, I., Tijan, R., and Rubin, G.M. 1994. The activities of two Ets-related transcription factors required for Drosophila eye development are modulated by the Ras/ MAPK pathway. Cell 78: 137-147.

Preston, C.R., Sved, J.A., and Engels, W.R. 1996. Flanking duplications and deletions associated with $P$-induced male recombination in Drosophila. Genetics 144: 1623-1638.

Rempel, R.E., Saenz-Robles, M.T., Storms, R., Morham, S., Ishida, S., Engel, A., Jakoi, L., Melhem, M.F., Pipas, J.M., Smith, C., et al. 2000. Loss of E2F4 activity leads to abnormal development of multiple cellular lineages. Mol. Cell 6: 293-306.

Ross, J.F., Liu, X., and Dynlacht, B.D. 1999. Mechanism of transcriptional repression of E2F by the retinoblastoma tumor suppressor protein. Mol. Cell 3: 195-205.

Ross, J.F., Naar, A., Cam, H., Gregory, R., and Dynlacht, B.D. 2001. Active repression and E2F inhibition by pRB are biochemically distinguishable. Genes \& Dev. 15: 392-397.

Royzman, I., Whittaker, A.J., and Orr-Weaver, T.L. 1997. Mutations in Drosophila DP and E2F distinguish G1-S progression from an associated transcriptional program. Genes \& Dev. 11: 1999-2011.

Royzman, I., Austin, R.J., Bosco, G., Bell, S.P., and Orr-Weaver, T.L. 1999. ORC localization in Drosophila follicle cells and the effects of mutations in $d E 2 F$ and $d D P$. Genes \& Dev. 13: $827-840$.

Rubin, G.M., Hong, L., Brokstein, P., Evans-Holm, M., Frise. E., Stapleton, M., and Harvey, D.A. 2000. A Drosophila complementary DNA resource. Science 287: 2222-2224.

Sambrook, J., Fritsch, E.F., and Maniatis, T. 1989. Molecular cloning: A laboratory manual. Cold Spring Harbor Laboratory Press, Cold Spring Harbor, NY.

Sardet, C., Vidal, M., Cobrinik, D., Geng, Y., Onufryk, C., Chen, A., and Weinberg, R.A. 1995. E2F-4 and E2F-5, two novel members of the E2F family, are expressed in the early phases of the cell cycle. Proc. Nat1. Acad. Sci. 92: 2403-2407.

Sawado, T., Hirose, F., Takahashi, Y., Sasaki, T., Shinomiya, T., Sakaguchi, K., Matsukage, A., and Yamaguchi, M. 1998a. The DNA replication-related element (DRE)/DRE-binding factor system is a transcriptional regulator of the Drosophila E2F gene. J. Biol. Chem. 273: 26042-26051.

Sawado, T., Yamaguchi, M., Nishimoto, Y., Ohno, K., Sakaguchi, K., and Matsukage, A. 1998b. dE2F2, a novel E2F-family transcription factor in Drosophila melanogaster. Biochem. Biophys. Res. Commun. 251: 409-415.

Sellers, W.R., Rodgers, J.W., and Kaelin, Jr., W.G. 1995. A potent transrepression domain in the retinoblastoma protein induces a cell cycle arrest when bound to E2F sites. Proc. Natl. 
Frolov et al.

Acad. Sci. 92: 11544-11548.

Takahashi, Y., Rayman, J., and Dynlacht, B. 2000. Analysis of promoter binding by the $\mathrm{E} 2 \mathrm{~F}$ and $\mathrm{pRB}$ families in vivo: Distinct E2F proteins mediate activation and repression. Genes \& Dev. 14: 804-816.

Verona, R., Moberg, K., Estes, S., Starz, M., Vernon, J.P., and Lees, J.A. 1997. E2F Activity is regulated by cell-cycle-dependent changes in subcellular localization. Mol. Cell Biol. 17: 7268-7282.

Wade, M., Kowalik, T.F., Mudryi, M., Huang, E.S., and Azizkhan, J.C. 1992. E2F mediates dihydrofolate reductase promoter activation and multiprotein complex formation in human cytomegalovirus infection. Mol. Cell. Biol. 12: 4364 4374.

Wells, J., Boyd, K., Fry, C., Bartley, S., and Farnham, P. 2000. Target gene specificity of E2F and pocket protein family members in living cells. Mol. Cell. Biol. 20: 5797-5807.

Wu, C.-L., Classon, M., Dyson, N., and Harlow, E. 1996. Expression of dominant-negative mutant DP-1 blocks cell cycle progression in G1. Mol. Cell. Biol. 16: 3698-3706.

Yamaguchi, M., Hayashi, Y., and Matsukage, A. 1995. Essential role of E2F recognition sites in regulation of the proliferating cell nuclear antigen gene promoter during Drosophila development. J. Biol. Chem. 270: 25159-25165.

Yamaguchi, M., Hirose, F., and Matsukage, A. 1996. Roles of multiple promoter elements of the proliferating cell nuclear antigen gene during Drosophila development. Genes Cells 1996 1: 47-58.

Yamaguchi, M., Hayashi, Y., Hirose, F., Nishimoto, Y., and Matsukage, A. 1997. Distinct roles of E2F recognition sites as positive or negative elements in regulation of the DNA polymerase alpha $180 \mathrm{kDa}$ catalytic subunit gene promoter during Drosophila development. Nucleic Acids Res. 25: $3847-3854$. 


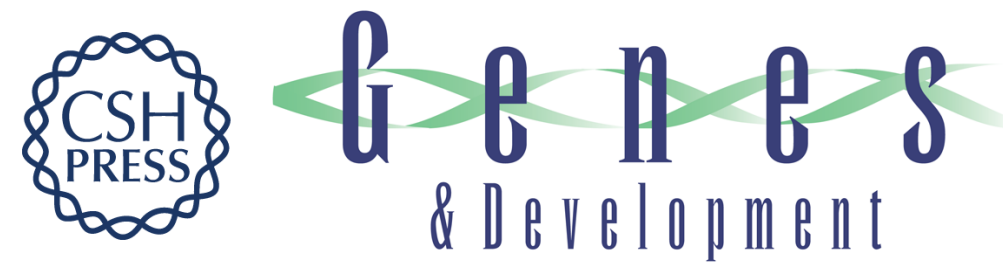

\section{Functional antagonism between E2F family members}

Maxim V. Frolov, David S. Huen, Olivier Stevaux, et al.

Genes Dev. 2001, 15:

Access the most recent version at doi:10.1101/gad.903901

References This article cites 73 articles, 46 of which can be accessed free at: http://genesdev.cshlp.org/content/15/16/2146.full.html\#ref-list-1

License

Email Alerting Receive free email alerts when new articles cite this article - sign up in the box at the top Service right corner of the article or click here.

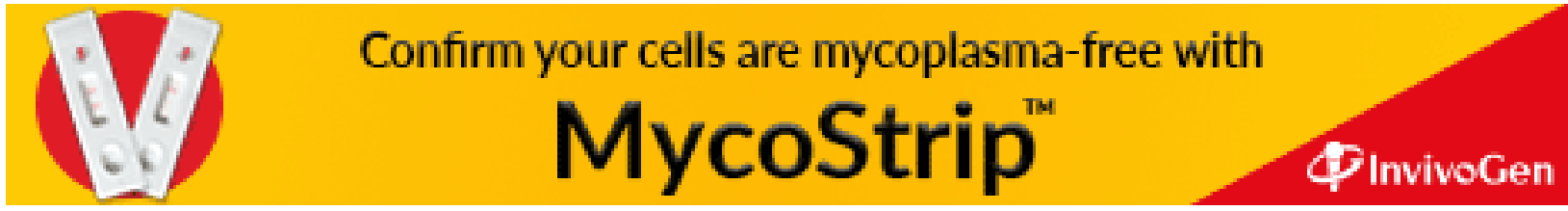

